\title{
Approximation Schemes for Independent Set and Sparse Subsets of Polygons
}

\author{
ANNA ADAMASZEK, University of Copenhagen \\ SARIEL HAR-PELED, University of Illinois Urbana-Champaign \\ ANDREAS WIESE, Universidad de Chile
}

We present a $(1+\varepsilon)$-approximation algorithm with quasi-polynomial running time for computing a maximum weight independent set of polygons from a given set of polygons in the plane. Contrasting this, the best-known polynomial time algorithm for the problem has an approximation ratio of $n^{\varepsilon}$. Surprisingly, we can extend the algorithm to the problem of computing the maximum cardinality subset of the given set of polygons whose intersection graph fulfills some sparsity condition. For example, we show that one can approximate the maximum subset of polygons such that the intersection graph of the subset is planar or does not contain a cycle of length 4 (i.e., $K_{2,2}$ ). Our algorithm relies on a recursive partitioning scheme, whose backbone is the existence of balanced cuts with small complexity that intersect polygons from the optimal solution of a small total weight.

For the case of large axis-parallel rectangles, we provide a polynomial time $(1+\varepsilon)$-approximation for the maximum weight independent set. Specifically, we consider the problem where each rectangle has one edge whose length is at least a constant fraction of the length of the corresponding edge of the bounding box of all the input elements. This is now the most general case for which a PTAS is known, and it requires a new and involved partitioning scheme, which should be of independent interest.

\section{CCS Concepts: • Mathematics of computing $\rightarrow$ Discrete mathematics; Graph theory; Approximation algorithms; • Theory of computation $\rightarrow$ Randomness, geometry and discrete structures; Compu- tational geometry;}

Additional Key Words and Phrases: Approximation algorithms, independent set, rectangles, approximation schemes

\section{ACM Reference format:}

Anna Adamaszek, Sariel Har-Peled, and Andreas Wiese. 2019. Approximation Schemes for Independent Set and Sparse Subsets of Polygons. F. ACM 66, 4, Article 29 (June 2019), 40 pages.

https://doi.org/10.1145/3326122

Anna Adamaszek supported by the Danish Council for Independent Research DFF-MOBILEX mobility grant.

Work on this article Sariel Har-Peled was partially supported by a NSF AF award CCF-1217462.

Authors' addresses: A. Adamaszek, Department of Computer Science, University of Copenhagen, Universitetsparken 1, DK-2100 Copenhagen, Denmark; email: anna@mpi-inf.mpg.de; S. Har-Peled, 201 N. Goodwin Avenue, Urbana, IL 618012302, USA; email: sariel@illinois.edu; A. Wiese (corresponding author), Departamento de Ingeniería Industrial, Facultad de Ciencias Físicas y Matemáticas, Universidad de Chile, Beauchef 851 Of. 705 Piso 7, Santiago Centro, Chile; email: awiese@dii.uchile.cl.

Permission to make digital or hard copies of all or part of this work for personal or classroom use is granted without fee provided that copies are not made or distributed for profit or commercial advantage and that copies bear this notice and the full citation on the first page. Copyrights for components of this work owned by others than the author(s) must be honored. Abstracting with credit is permitted. To copy otherwise, or republish, to post on servers or to redistribute to lists, requires prior specific permission and/or a fee. Request permissions from permissions@acm.org.

(C) 2019 Copyright held by the owner/author(s). Publication rights licensed to ACM.

0004-5411/2019/06-ART29 \$15.00

https://doi.org/10.1145/3326122 


\section{INTRODUCTION}

In this article, we study the Independent Set of Polygons problem. We are given a set $\mathcal{P}=$ $\left\{\sigma_{1}, \ldots, \sigma_{m}\right\}$ of $m$ simple polygons in the plane, with weights $w_{1}, w_{2}, \ldots, w_{m}>0$, respectively, encoded by $n$ input bits. Our goal is to find an independent set of polygons from $\mathcal{P}$ of a maximum total weight. A set of polygons is independent if no two polygons from the set intersect, where we treat polygons as open sets.

This problem and its special cases arise in various settings, such as (i) channel admission control [39], (ii) chip manufacturing [34], (iii) map labeling [2, 22, 51], (iv) cellular networks [14], (v) unsplittable flow $[1,12]$, (vi) data mining $[25,38,40]$, and many others.

A natural approach to this problem is to build an intersection graph $\mathrm{G}=(\mathrm{V}, \mathrm{E})$, where we have one vertex for each input polygon and two vertices are connected by an edge if and only if their corresponding polygons intersect. The weight of each vertex equals the weight of its corresponding polygon. The task at hand is to compute the maximum weight independent set in G. In general graphs, even the unweighted maximum independent set problem does not allow an approximation factor within $|\mathrm{V}|^{1-\varepsilon}$ for any $\varepsilon>0$, if $N \mathrm{P} \neq \mathrm{P}$ [52]. Surprisingly, even if the maximum degree of the graph is bounded by 3, no PTAS is possible [10] (assuming that $N \mathrm{P} \neq \mathrm{P}$ ). However, in our case the intersection graph stems from geometric objects, and we can make use of the exact locations of the input polygons in our computations. As we demonstrate, this allows obtaining much better approximation factors.

Fat (convex) polygons. If the input objects are fat (e.g., disks or squares), then PTASes are known. One approach $[19,24]$ relies on a hierarchical spatial subdivision, such as a quadtree, combined with dynamic programming techniques [4]. This approach works even in the weighted case. Another approach [19] relies on a recursive application of a nontrivial generalization of the planar separator theorem $[41,50]$. However, this approach is limited to the unweighted case.

Axis-parallel rectangles. The problem turns out to be significantly harder already for the setting of axis-parallel rectangles. No constant factor approximation algorithms are known in this setting, while the best-known hardness result is strong NP-hardness [28, 36]. This gap remains despite a lot of research on the problem $[2,9,13,17,20,28,36,38,39,46]$. For the weighted case, there are several $O(\log m)$ approximation algorithms known [2, 38, 46], and the hidden constant can be made arbitrarily small, since for any constant $k$ there is a $\left\lceil\log _{k} m\right\rceil$-approximation algorithm due to Berman et al. [9]. Chan and Har-Peled [17] provided an $O(\log m / \log \log m)$ approximation for the weighted case. For the unweighted case, an $O(\log \log m)$-approximation was given by Chalermsook and Chuzhoy [13].

Some algorithms have been studied that perform better for special cases of the problem. There is a $4 q$-approximation algorithm due to Lewin-Eytan et al. [39] where $q$ denotes the size of the largest clique in the given instance. In case when the optimal independent set has size $\beta m$ for some $\beta \leq 1$, Agarwal and Mustafa present an algorithm that computes an independent set of size $\Omega\left(\beta^{2} m\right)[3]$.

Other input objects. For the case when the input instance is a collection of $m$ line segments, an $O\left(\left(m_{\text {opt }}\right)^{1 / 2+o(1)}\right)$-approximation was developed by Agarwal and Mustafa [3], where $m_{\text {opt }}$ is the size of the optimal solution. Fox and Pach [27] have improved the approximation factor to $m^{\varepsilon}$ for line segments, and also curves that intersect a constant number of times. Their argument relies on the intersection graph having a large biclique if it is dense, and a cheap separator otherwise.

For an independent set of unweighted pseudo-disks, Chan and Har-Peled [17] provided a PTAS. Surprisingly, their algorithm is a simple local search strategy that relies on using the planar separator theorem to argue that if the local solution is far from the optimal, then there is a "small" beneficial exchange. 
The challenge. Although the complexity of geometric independent set is well understood in the setting of squares, for axis-parallel rectangles the problem is still widely open. In particular, the techniques of the above approximation schemes for squares do not carry over to rectangles. The PTAS from Reference [24] requires that every horizontal or vertical line intersects only a bounded number of objects of the optimal solution that are relatively large in at least one dimension. For rectangles, this number can be up to $\Theta(m)$, which is too much. For the local search techniques, one can easily construct examples showing that for any size of exchanges (which still yields quasipolynomial running time), the optimum is missed by a factor of up to $\Omega\left(m /(\log m)^{O(1)}\right)$.

\subsection{Our Results}

We present the first $(1-\varepsilon)$-approximation algorithm to the problem of computing the maximum weight independent set of polygons, with a quasi-polynomial running time of $2^{\text {poly }(\log m, 1 / \varepsilon)}$. $n \log n$. In particular, our algorithm works for axis-parallel rectangles, line segments, and arbitrary polygons. As mentioned above, the best-known polynomial time approximation algorithm for our setting has a ratio of $m^{\varepsilon}$ [27], and even for axis-parallel rectangles the currently best-known ratios are $O(\log m / \log \log m)$ for the weighted case [17], and $O(\log \log m)$ for the unweighted case [13].

We are not aware of any previous algorithms for the problem with quasi-polynomial running time that would give better bounds than the above-mentioned polynomial time algorithms. Our QPTAS rules out the possibility that the problem is APX-Hard, assuming that NP $\nsubseteq$ $\operatorname{DTIME}\left(2^{\text {polylog(n)}}\right)$, and thus it suggests that it should be possible to obtain significantly better polynomial time approximation algorithms for the problem $^{1}$.

Then we show how to extend our QPTAS to computing subsets of polygons whose intersection graph complies with a given sparsity property. In addition, we present a PTAS for the case of $\delta$-large rectangles for any constant $\delta>0$, i.e., for the case when each input rectangle has at least one edge of length at least $\delta N$, assuming that in the input only integer coordinates within $\{0, \ldots, N\}$ occur.

We give an overview for the previous and the new results for the problem in Figure 1.

\subsection{Technical Contribution}

Recursive partitioning. The key technique in our QPTAS is a new geometric partitioning scheme. We prove that for the polygons in the optimal solution (and for any set of non-intersecting polygons) there exists a balanced cut that intersects only a weighted $O(\varepsilon / \log m)$-fraction of the polygons, and that can be described by only $O(\operatorname{poly}(\log m, 1 / \varepsilon))$ bits. Due to the latter property there are only quasi-polynomially many candidates for this cut, and thus we can try all of them in quasipolynomial time. The polygons intersecting the cut are "lost," as the algorithm throws them away. Then the algorithm calls itself recursively on both sides of the cut until we obtain subproblems (described by subparts of the input area) that contain only a few polygons from the optimal solution, which can be solved directly by brute force. Since the cuts are balanced, the recursion depth is $O(\log m)$, and thus the overall running time of the algorithm is quasi-polynomial.

Cheap balanced cuts. To show that a cut with the claimed property always exists, we use cuttings $[11,18]$, which yields a planar graph, where each face intersects a relatively small fraction of the optimal solution. We then use a separator theorem for planar graphs, applied to the cuttings, to get a cheap balanced partition of the area into two pieces. To the best of our knowledge, the idea of

\footnotetext{
${ }^{1}$ Indeed, if a problem is APX-Hard, then a QPTAS for it would imply that 3-SAT can be solved in $2^{\text {polylog }(n)}$ time, which is unlikely. Furthermore, the exponential time hypothesis $(\mathrm{ETH})$, which is believed to be true, states that 3-SAT cannot be solved in time better than $2^{c n}$ for some absolute constant $c$. Therefore, a QPTAS for an APX-Hard problem would refute the ETH.
} 


\begin{tabular}{|c|c|c|c|c|}
\hline \multicolumn{5}{|c|}{ Independent set } \\
\hline Shape & Attributes & approximation & Ref & running time \\
\hline \multirow{3}{*}{$\begin{array}{l}\text { Axıs-paraīiei } \\
\text { rectangles }\end{array}$} & Unweighted & $O(\log \log m)$ & [CC09] & \multirow{2}{*}{$m^{O(1)}$} \\
\hline & Weighted & $O\left(\frac{\log m}{\log \log m}\right)$ & [CH12] & \\
\hline & $\begin{array}{l}\delta \text {-large weighted } \\
\text { rectangles with } \\
\text { vertices in } \llbracket N \rrbracket^{2} \text {. }\end{array}$ & $1+\varepsilon$ & Theorem $4.23_{\mathrm{p} 35}$ & $(m N)^{1 /(\varepsilon \delta)^{O(1)}}$ \\
\hline Segs/curves & $\begin{array}{l}\text { Unweighted and at most } \\
k=O(1) \\
\text { intersection points per } \\
\text { pair of curves. }\end{array}$ & $m^{\varepsilon}$ & [FP11] & $n^{O\left((4 / \varepsilon)^{-2 / \varepsilon}\right)}$ \\
\hline $\begin{array}{l}\text { Segs / curves } \\
\text { rects / polygons }\end{array}$ & Weighted & $1+\varepsilon$ & Theorem $2.4 \mathrm{p} 6$ & $2^{\text {poly }(\log m, 1 / \varepsilon)} \cdot n \log n$ \\
\hline \multicolumn{5}{|c|}{ Sparse properties } \\
\hline Polygons & $\begin{array}{l}\text { Unweighted, pairs inter- } \\
\text { sect } O(1) \\
\text { times }\end{array}$ & $1+\varepsilon$ & Theorem $3.20_{\mathrm{p} 18}$ & $2^{\text {poly }(\log m, 1 / \varepsilon)} \cdot n^{O(1)}$ \\
\hline
\end{tabular}

Fig. 1. Summary of known and new results. Here $\llbracket N \rrbracket=\{1, \ldots, N\}$.

using planar separators together with cuttings is novel and is one of the key contributions of this work. Since the input polygons are weighted, we need weighted cuttings, and while this is an easy extension of known techniques, this is not written explicitly in detail anywhere. As such, for the sake of self-containment, we reprove here the weighted version of the exponential decay lemma of Chazelle and Friedman [16]. Our proof seems to be simpler than the previous proofs, and the constants are slightly better, and as such the result might be of independent interest.

Extensions to other sparsity conditions. When we ask for an independent set of the input polygons, we require that the intersection graph corresponding to the set of computed polygons contains only isolated vertices. Such a graph is the ultimate sparse graph. Using the new approach we can also obtain a QPTAS when other, more relaxed sparsity conditions are required from the intersection graph of the computed polygons. For technical reasons, here we need to assume that every pair of input polygons intersects a constant number of times (note that we did not need this assumption in the independent set case) and that the input polygons are unweighted. We provide a QPTAS for any sparsity condition that guarantees that the intersection graph corresponding to the set of computed objects has a sub-quadratic number of edges. There are many conditions that fall in this category, for instance, that the intersection graph is planar or that it does not contain a $K_{s, t}$ as a subgraph, where $s, t$ are some constants. If the input polygons are pseudo-disks, then we can even compute the maximum set such that no point in the plane is covered by more than $d$ polygons, for any given constant $d$.

PTAS for large rectangles. When the input instance is a set of axis-parallel rectangles where each rectangle has at least one large edge compared to the length of the corresponding edge of the bounding box, we provide a different partitioning scheme that leads to a PTAS. It requires a novel and rather involved construction, as we cannot use the standard tools to facilitate it. Our partition has two levels. At the top level, we partition the plane into a constant number of polygons with constant complexity each. Some of the polygons of the partition correspond to a single (potentially large) rectangle, and the others are narrow corridors with constant complexity each. This step incurs only a small loss, and the number of possible partitions is polynomial, so our algorithm 
can try all of them. In the second level, we show how to decompose each narrow polygon of the partition recursively in a way that is dynamic programming (DP) friendly. Then we can use the DP to find a near-optimal solution in polynomial time. We believe that this new partition scheme and the associated dynamic programming algorithm are the first step in obtaining a PTAS for the general problem.

\subsection{Impact of This Work}

This article contains two new technical concepts: the cheap balanced (geometric) cuts, used for our QPTASs, and the partition of the plane into few large rectangles and narrow corridors, used for the PTAS for $\delta$-large rectangles. Following the initial conference publication of this work [5, 6], both techniques have been used to obtain other results for a variety of geometric problems.

Using the cheap balanced cuts, Mustafa et al. [45] showed that one can get a QPTAS for geometric set cover of points by weighted pseudodisks. Since the problem becomes APX-Hard for fat triangles of similar size [30], this is the best one can hope for. This demonstrates that the geometric set cover and geometric independent set problems in the plane are inherently different with respect to approximability. Bandyapadhyay et al. [8] used cheap balanced cuts for designing QPTASs for the convex decomposition problem and the surface approximation problem. Marx and Pilipczuk [44] used them to find faster algorithms for facility location problems on planar graphs and in the two-dimensional plane. Also, Karpinski et al. [37] used them to approximate the base of the number of triangulations of a planar point set.

The partition into a constant number of corridors has been used by Adamaszek and Wiese [7] as a starting point to get a QPTAS for the geometric two-dimensional knapsack problem. They refined the corridor partition further to a partition into a poly-logarithmic number of rectangular boxes that separates the rectangles that are large in the horizontal dimension from those that are large in the vertical dimension. Moreover, Nadiradze and Wiese [47] used the corridor partition to obtain a $(1.4+\varepsilon)$-approximation algorithm in pseudo-polynomial time for the strip-packing problem. Here, also, the partition into corridors was used as a starting point to a more refined partition into a constant number of rectangular boxes. The corridor decomposition was also used by Har-Peled [33] as a tool in designing a sublinear space algorithm for shortest path in a polygon.

Paper organization. In Section 2, we describe the QPTAS for the maximum weight independent set of polygons, where the low-level decomposition tools needed for the algorithm are described in Section 3. Specifically, in Section 3.1, we describe a canonical decomposition of the complement of the union of disjoint polygons, and we show how to extend it to work for arbitrary intersecting polygons. In Section 3.2 we reprove the exponential decay lemma, show how to build weak $1 / r$-cuttings of disjoint polygons of size $O(r \log r)$, and spell out the conditions enabling one to compute smaller $1 / r$-cuttings of size $O(r)$. In Section 3.4, we describe the extension to a QPTAS for computing the maximum sparse subset of polygons. In Section 4, we describe the PTAS for large axis-parallel rectangles. We conclude in Section 5 with some comments.

\section{A QPTAS FOR INDEPENDENT SET OF POLYGONS}

In this section, we present our $(1-\varepsilon)$-approximation algorithm for the problem of computing a maximum weight independent set of polygons with a quasi-polynomial running time.

An instance of the problem consists of a set of $m$ weighted simple polygons $\mathcal{P}=\left\{\sigma_{1}, \ldots, \sigma_{m}\right\}$, with a total of $n$ vertices. Let $\varepsilon>0$ be a fixed approximation parameter. First, we ensure that the weights of the input polygons are integers in a polynomial range without changing the instance significantly, by losing at most a factor of $1-\varepsilon$ in the weight of an optimal solution $O$. Observe that the following lemma implies that $w(O) \leq m^{2} / \varepsilon$. 
Lemma 2.1. If there is a (quasi-)polynomial time $(1+\varepsilon)$-approximation algorithm for the case that $w(\sigma) \in \llbracket m / \varepsilon \rrbracket=\{1, \ldots, m / \varepsilon\}$, for each polygon $\sigma \in \mathcal{P}$, then there is a (quasi-)polynomial time $(1+\varepsilon)^{2}$-approximation algorithm for the general case.

Proof. We scale the weights of all polygons such that $\gamma=\max _{\sigma \in \mathcal{P}} w(\sigma)=m / \varepsilon$. For the weight $w(O)$ of the optimal solution $O$, we have that $w(O) \geq \gamma \geq m / \varepsilon$. It follows that rounding down the weight of each polygon to the closest integer costs at most $m$ overall, which is at most $\varepsilon w(O)$. Polygons of weight zero after the rounding can be removed.

From this point on, we assume that $w(\sigma) \in \llbracket m / \varepsilon \rrbracket$ for each polygon $\sigma \in \mathcal{P}$. The key ingredient of the new algorithm is that for any independent set of polygons $\mathcal{P}^{\prime} \subseteq \mathcal{P}$, and in particular for the optimal solution, there exists a cheap balanced cut.

Definition 2.2. Given a set $\mathcal{P}^{\prime} \subseteq \mathcal{P}$ of polygons in the plane, a cheap balanced cut is a polygon $\Gamma$, with the following three properties:

(A) the total weight of polygons in $\mathcal{P}^{\prime}$ that are intersected by $\Gamma$ is at most $\frac{\varepsilon}{\log m} w\left(\mathcal{P}^{\prime}\right)$,

(B) the total weight of polygons in $\mathcal{P}^{\prime}$ that are completely inside (respectively, outside) of $\Gamma$ is at most $(9 / 10) w\left(\mathcal{P}^{\prime}\right)$,

(C) the polygon $\Gamma$ can be fully encoded by a binary string with poly $(\log m, 1 / \varepsilon)$ bits, and

(D) the polygon $\Gamma$ has $O(n)$ vertices.

Lemma 2.3. For any independent set of polygons $\mathcal{P}^{\prime} \subseteq \mathcal{P}$ there exists a cheap balanced cut $\Gamma$ or there is a polygon $\sigma \in \mathcal{P}^{\prime}$ such that $w(\sigma) \geq w\left(\mathcal{P}^{\prime}\right) / 10$.

The proof of Lemma 2.3 is in Section 3 (see Remark 3.15). Our algorithm enumerates all polygons $\Gamma$ that could be cheap balanced cuts corresponding to the (unknown) optimal solution $O \subseteq \mathcal{P}$. Since the encoding of such a polygon is short, by Definition $2.2(\mathrm{C})$, there are at most $2^{\text {poly }(\log m, 1 / \varepsilon)}$ such polygons, and we can enumerate all of them in quasi-polynomial time. For each enumerated cut $\Gamma$ we call recursively on two subproblems. One subproblem consists of all input polygons that lie completely inside $\Gamma$, the other consists of all input polygons that lie completely outside of $\Gamma$. We solve these subproblems recursively and combine the obtained solutions into a global solution to the original problem.

A degenerate case here is that the optimal solution $O^{\prime}$, for the current subproblem $\mathcal{P}^{\prime}$, contains a polygon $\sigma \in O^{\prime}$ such that $w(\sigma) \geq \frac{2}{3} w\left(O^{\prime}\right)$. In the case, the cut is defined by $\sigma$ - one subproblem is $\{\sigma\}$, and the other subproblem is the set of all the polygons in $\mathcal{P}^{\prime}$ that do not intersect $\sigma$.

If at each step the algorithm correctly guesses the cheap balanced cut $\Gamma$, then the recursion has a depth of $O(\log w(O))=O(\log (m / \varepsilon))$. Therefore, the algorithm stops the recursion after $O(\log (m / \varepsilon))$ levels (naturally, the algorithm also returns immediately if the given subproblem is empty).

Running time. In each node of the recursion tree the algorithm enumerates at most $2^{\alpha}$ candidates for the cheap balanced cut $\Gamma$, where $\alpha=\operatorname{poly}(\log m, 1 / \varepsilon)$. Thus, each node has at most $2^{\alpha}$ children. Now for each cut $\Gamma$, the algorithm partitions the polygons from the current input instance into three groups:

(i) polygons intersecting $\Gamma$,

(ii) polygons contained in the interior of $\Gamma$, and

(iii) polygons contained in the exterior of $\Gamma$.

The cut polygon $\Gamma$ has $O(n)$ vertices, and this partition can be computed in $n^{O(1)}$ time-and in $O(n \log n)$ time if one is more careful the implementation, see Remark 3.17 below. The algorithm then call recursively on the two subproblems defined by the partition. The recursion depth 
is $h=O(\log (m / \varepsilon))$. As such, a recursive subproblem is encoded by $\beta=O(h \operatorname{poly}(\log m, 1 / \varepsilon))=$ poly $(\log m, 1 / \varepsilon)$ bits, and thus there are $2^{\beta}$ different subproblems overall. The overall overall running time is $2^{\beta} 2^{\alpha} O(n \log n)=2^{\text {poly }(\log m, 1 / \varepsilon)} O(n \log n)$.

Approximation ratio. For the correct sequence of cuts, at each level of the recursion the weight of an optimal solution changes at most by a factor of $1-\frac{\varepsilon}{\log m}$, since a cheap balanced cut intersects polygons whose total weight is at most a $\frac{\varepsilon}{\log m}$-fraction of the optimal solution of the respective subproblem. Thus, the obtained approximation ratio is at least $\left(1-\frac{\varepsilon}{\log m}\right)^{O(\log (m / \varepsilon))}=1-O(\varepsilon)$.

We thus obtain the following.

Theorem 2.4. Let $\mathcal{P}=\left\{\sigma_{1}, \ldots, \sigma_{m}\right\}$ be a set of $m$ simple polygons in the plane, with $n$ vertices, where $\sigma_{i}$ has weight $w\left(\sigma_{i}\right)$, for $i=1, \ldots, m$. Then an independent set $\mathcal{P}^{\prime} \subseteq \mathcal{P}$ of weight at least $(1-\varepsilon) w(O)$ can be computed in time $2^{\text {poly }(\log m, 1 / \varepsilon)} n \log n$, where $O$ is the optimal solution.

\section{DECOMPOSITIONS AND CUTTINGS}

Our goal in this section is to prove Lemma 2.3-to show that for any independent set of polygons there exists a cheap balanced cut. We show a stronger constructive result by providing an algorithm that for a given set of non-overlapping polygons computes a cheap balanced cut efficiently. Of course, in our settings, the set of these non-overlapping polygons that form the optimal solution is not known, so only the existence of the cheap balanced cut is used in the analysis of the algorithm of Section 2.

First, in Section 3.1, we describe a canonical decomposition for the plane, guided by the polygons of the optimal solution. Based on this, in Section 3.2 we show that there is an $1 / r$-cutting with small complexity. Finally, in Section Section 3.3 we prove Lemma 2.3.

Later, in Section 3.4 we extend our reasoning for independent set to more general settings. Those involve in particular cases in which the optimal solution consists of polygons that might overlap. Therefore, we present our reasoning about cuttings also for the case of polygons that might intersect.

Remark 3.1. The reader might benefit from thinking about the polygons as just being line segments. The corridor decomposition is vertical decomposition in this case, and the needed cuttings are already known for this case.

\subsection{Decomposing an Arrangement of Polygons into Corridors}

3.1.1 A Canonical Decomposition for Disjoint Polygons. Let $\mathcal{P}=\left\{\sigma_{1}, \ldots, \sigma_{m}\right\}$ be a set of $m$ nonoverlapping simple polygons in the plane, of total complexity $n$. We also have a special outside square that contains all the polygons of $\mathcal{P}$, which is the frame. For the sake of simplicity of exposition, we assume that all the edges involved in $\mathcal{P}$ and the frame are neither horizontal nor vertical. This can be ensured by slightly rotating the axis system. ${ }^{2}$

We are interested in a canonical decomposition of the complement of the union of the polygons of $\mathcal{P}$ inside the frame into corridors, that has the property that the number of corridors is $O(m)$, and every corridor is defined by a constant number of polygons of $\mathcal{P}$. See Figure 2 for an example. Consider the medial axis of $\mathcal{P}$. To make the presentation easier ${ }^{3}$, we use the $L_{\infty}$-medial axis $\mathcal{M}=\mathcal{M}(\mathcal{P})$. Specifically, a point $p \in \mathbb{R}^{2}$ is in $\mathcal{M}$ if there is an $L_{\infty}$-ball (i.e., an axis-parallel square $\square$ centered in $p$ ) that touches the polygons of $\mathcal{P}$ or the frame in two or more points, and the interior

\footnotetext{
${ }^{2}$ In the example of Figure 2, we do not bother to do this, and the frame is axis-parallel.

${ }^{3} \mathrm{Or}$ at least to make the drawing of the figures easier.
} 


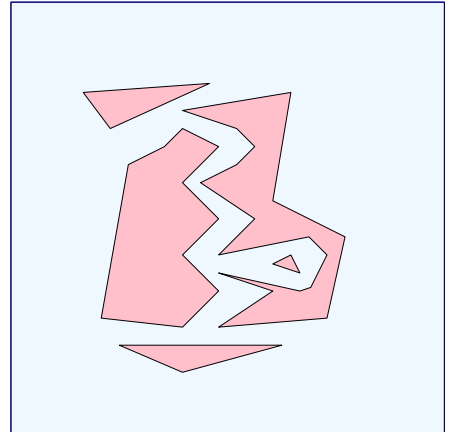

(A) The polygons of $\mathcal{P}$, and the frame.

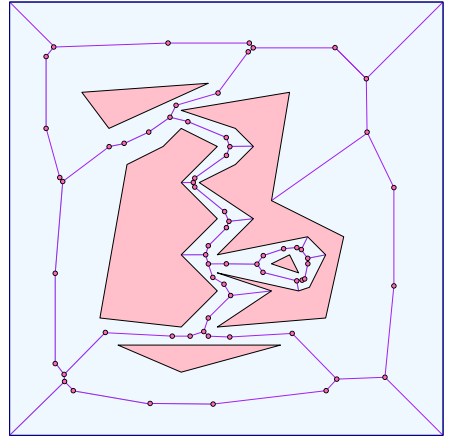

(C) $\mathcal{M}$ : The $L_{\infty}$ medial axis.

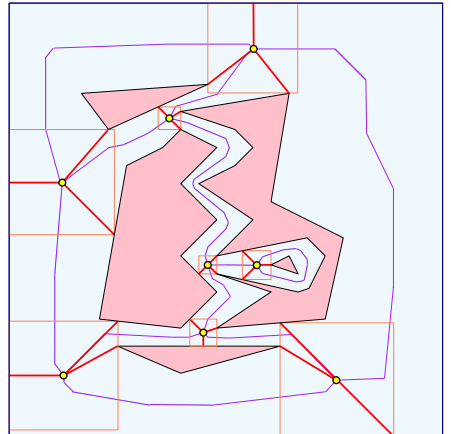

(E) The vertices of degree 3, their critical squares, and the spokes they induce.

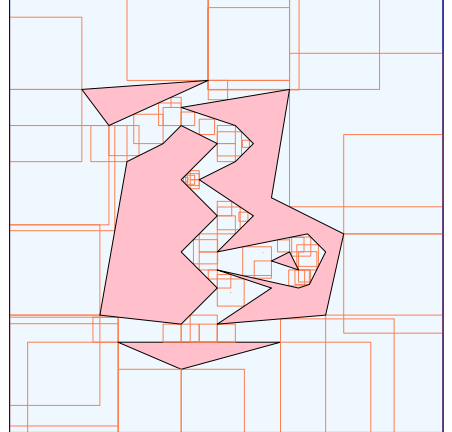

(B) Some critical squares.

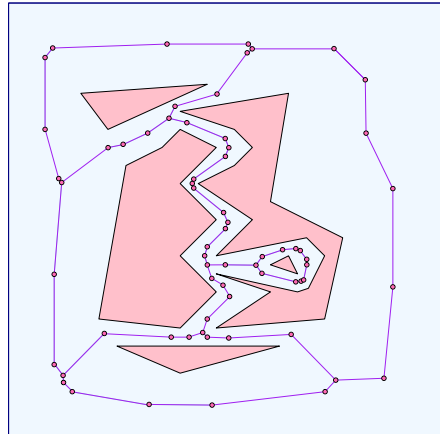

(D) $\mathcal{M}^{\prime}$ : The reduced $L_{\infty}$ medial axis.

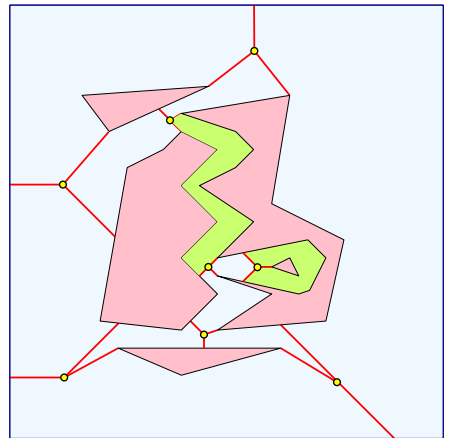

(F) The resulting corridor decomposition, and some corridors.

Fig. 2. Building up the corridor decomposition.

of $\square$ does not intersect any of the polygons of $\mathcal{P}$ or the exterior of the frame. We refer to a square $\square$ with the above properties as a critical square.

The $L_{\infty}$-medial axis is a connected collection of interior disjoint segments (i.e., it is the boundary of the Voronoi diagram of the polygons in $\mathcal{P}$ under the $L_{\infty}$ metric together with some extra bridges involving points of the medial axis that have the same polygon on both sides). The medial axis $\mathcal{M}$ contains some features that are of no use to us-specifically, we repeatedly remove vertices of degree one in $\mathcal{M}$ and the segments that support them-this process removes unnecessary tendrils. 
Let $\mathcal{M}^{\prime}$ be the resulting structure after this cleanup process. Note that this is exactly the boundary of the Voronoi diagram of the input polygons.

Let $V=V\left(\mathcal{M}^{\prime}\right)$ be the set of vertices of $\mathcal{M}^{\prime}$ of degree at least three. Each such vertex corresponds to a point $p \in \mathbb{R}^{2}$, which has a critical square $\square_{\mathrm{p}}$ associated with it. For such a square $\square_{\mathrm{p}}$, there are $k \geq 3$ input polygons (not necessarily pairwise distinct) that it touches, and let $\mathrm{p}_{1}, \ldots, \mathrm{p}_{k}$ be these $k$ points of contact (under general position assumption $k$ would be 3 ). We refer to the segments $\mathrm{pp}_{1}, \mathrm{pp}_{2}, \ldots, \mathrm{pp}_{k}$ as the spokes of $\mathrm{p}$. Since no edge of the input polygons or the frame is axis parallel, the spokes are uniquely defined.

Let $\mathrm{S}$ be the set of all spokes defined by the vertices of V. Consider the arrangement formed by the polygons of $\mathcal{P}$ together with the segments of $\mathrm{S}$. This decomposes the complement of the union of $\mathcal{P}$ contained inside the frame into polygons. Each such polygon boundary is made from two polygonal chains that lie on two polygons of $\mathcal{P}$ and four spokes. In particular, each such polygon is a simple polygon. We refer to such a polygon as a corridor, and we denote by $\mathcal{C}(\mathcal{P})$ the collection of corridors corresponding to $\mathcal{P}$. The set of corridors $\mathcal{C}(\mathcal{P})$ is the corridor decomposition of $\mathcal{P}$.

Definition 3.2. Let $\mathcal{C}(\mathcal{P})$ be the corridor decomposition of a set of non-overlapping simple polygons $\mathcal{P}$ in the plane, and let $Q \subseteq \mathcal{P}$.

(A) Consider a corridor $C \in \mathcal{C}(Q)$. Then, there exists a subset $\mathcal{B} \subseteq \mathcal{P}$, of size at most 4 , such that $C \in \mathcal{C}(\mathcal{B})$. We denote the set $\mathcal{B}$ by $D(C)$ and call it a defining set of the corridor $C$.

(B) For a corridor $C \in \mathcal{C}(Q)$, a polygon $\sigma \in \mathcal{P}$ conflicts with $C$, if $C$ is not a corridor of $\mathcal{C}(D(C) \cup\{\sigma\})$. This happens if $\sigma$ intersects $C$, or alternatively, if the presence of $\sigma$ prevents the creation of the two vertices of the medial axis defining $C$. The set of polygons in $\mathcal{P} \backslash D(C)$ that conflict with $C$ is the stopping set (or conflict list) of $C$ and is denoted by $K(C)$.

Note that the defining set of a corridor might not be unique, and that a defining set might define several corridors. The stopping set is the same, independent of the choice of the defining set. Note also that if any pair of input polygons intersects only $O(1)$ times then any defining set of constant size can define only a constant number of corridors.

Lemma 3.3. For a set $\mathcal{P}$ of $m$ disjoint simply connected polygons (i.e., polygons without holes) in the plane, we have that $|\mathcal{C}(\mathcal{P})|=O(m)$.

Proof. Consider the reduced medial axis $\mathcal{M}^{\prime}$. It can be naturally interpreted as a connected planar graph, where the vertices of degree at least three form the vertex set $\mathrm{V}$, and two vertices are connected by an edge if there is a path $\pi$ on $\mathcal{M}^{\prime}$ that connects them, and there is no vertex of $\mathrm{V}$ in the interior of $\pi$. Let $\mathrm{G}=(\mathrm{V}, \mathrm{E})$ be the resulting graph.

Observe that the drawing of $\mathrm{G}$ has $m+1$ faces, as each face contains a single polygon of $\mathcal{P}$ in its interior (except for the outer face, which "contains" the frame). The graph G might contain both self loops and parallel edges. However, every vertex of $\mathrm{G}$ has degree at least 3 . As such, we have that $e \geq 3 v / 2$, where $v$ and $e$ are the number of vertices and edges in $\mathrm{G}$, respectively.

Euler's formula in this case states that $m+1-e+v=2$ (the formula holds even if the graph contains loops and parallel edges). As such we have that $m+1-(3 v / 2)+v \geq 2$, which implies that $2 m+2 \geq v+4$; that is $v \leq 2 m-2$. This in turn implies that $m+1-e+(2 m-2) \geq 2$, which implies that $e \leq 3 m-3$. Now, clearly, every corridor corresponds to one edge of G, which implies the claim.

3.1.2 A Canonical Decomposition for Intersecting Polygons. Let $Q=\left\{\sigma_{1}, \ldots, \sigma_{m}\right\}$ be a set of $m$ simple polygons in the plane of total complexity $n$, that are not necessarily disjoint. In the 


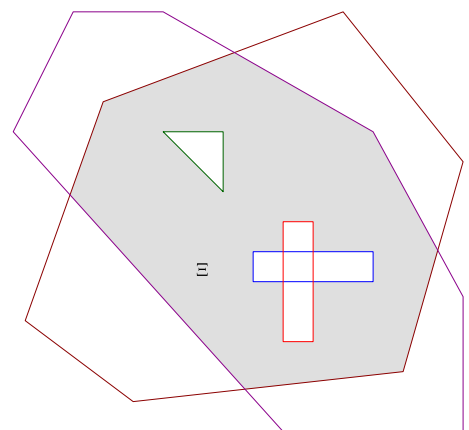

(A) The polygons defining face $\Xi$ (gray)

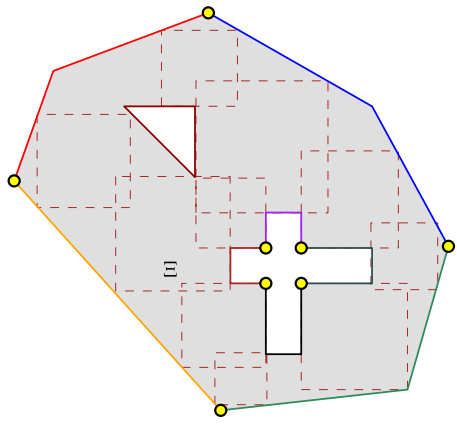

(C) The critical squares.

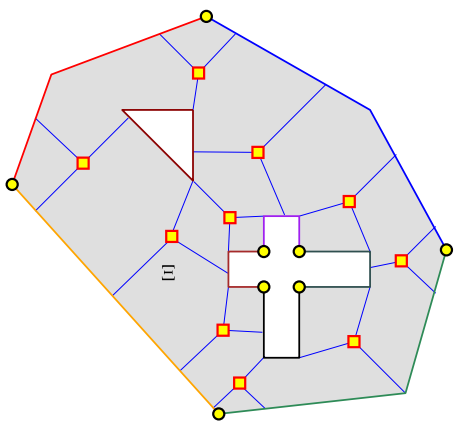

(E) The vertices of degree 3, and their spokes.

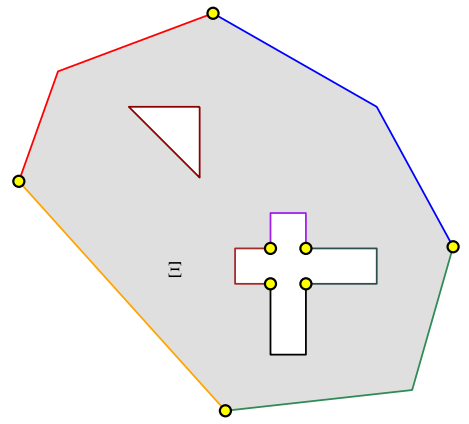

(B) The edges defining the boundary of $\Xi$.

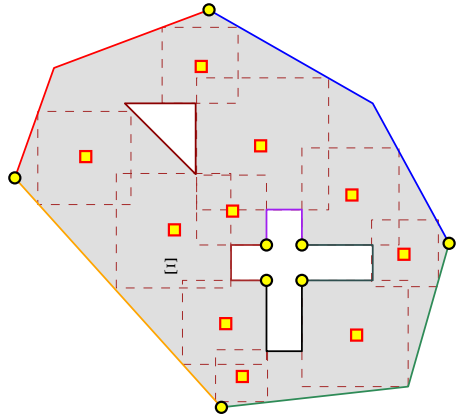

(D) The medial axis vertices generated.

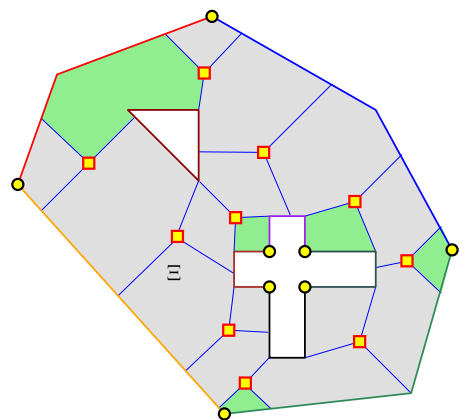

(F) The resulting corridor decomposition, and some corridors.

Fig. 3. Building up the corridor decomposition for a single face $\Xi$, for non-disjoint polygons.

following, we think about each polygon as being a (closed) curve, and we naturally assume that no three curves pass through a common point.

For two curves of $Q$, an intersection point of their boundaries is an intersection vertex. Consider the arrangement $\mathcal{A}(Q)$ - it is a decomposition of the plane into faces, i.e., maximal connected components that avoid the curves of $Q$. The maximum connected portion on a curve between two intersection vertices is an edge. If a curve has no intersection points on it, then the whole curve is an edge. See Figure 3(A) and (B) for an example.

Consider a face $\Xi$ of the arrangement $\mathcal{A}(Q)$. Denote by $t_{\Xi}$ the number of intersection vertices on the boundary of $\Xi$, and by $k_{\Xi}$ the number of connected components of the boundary of $\Xi$. Each 
connected component of the boundary can be broken into several edges. To decompose the face $\Xi$ into corridors, we apply a modified version of Lemma 3.3, where we treat the connected components of the boundary of $\Xi$ as polygons, and the outer connected component of the boundary as the frame. The main modification is that during the cleanup process, we do not delete the tendrils that rise from the intersection vertices (i.e., the endpoint of a medial axis edge ending at an intersection vertex is not deleted). ${ }^{4}$ Each such tendril would give rise to one additional corridor. An example of the resulting decomposition into corridors is depicted in Figure 3.

Repeating the above operation for all the faces in the arrangement $\mathcal{A}(Q)$ results in a decomposition of the whole plane into a collection of corridors $\mathcal{C}(Q)$. Now, unlike in the setting of disjoint input polygons, a corridor might be contained in the interior of several polygons of $Q$. However, we still have the property that no polygon boundary intersects the interior of a corridor.

Lemma 3.4. Let $Q$ be a set of $m$ simply connected polygons in the plane, let $\mathrm{t}$ be the total number of intersection vertices in $\mathcal{A}(Q)$, and let $\mathcal{C}(Q)$ be the corridor decomposition of $Q$. Then $|\mathcal{C}(Q)|=$ $O(m+\mathrm{t})$.

Proof. Observe that every intersection vertex can contribute to at most four faces. Similarly, a single curve such that there is no vertex on it, is on the boundary of two faces. Therefore, the total number of edges for all faces of the arrangement is $O(m+\mathrm{t})$, and we get $|\mathcal{C}(Q)|=O(m+\mathrm{t})$.

3.1.3 Loose and Tight Corridors. Let $\mathcal{P}$ be a set of $m$ polygons, where every pair of them intersects at most $\zeta$ times. Let $\mathcal{R}=\bigcup_{\mathcal{S} \subseteq \mathcal{P}} \mathcal{C}(\mathcal{S})$ be the set of all corridors that are induced by some subset of polygons of $\mathcal{P}$.

A corridor $C$ that is defined by a set $D=D(C)$ of polygons that are pairwise disjoint is a loose corridor. A corridor whose defining set contains a pair of intersecting polygons is tight.

LEMMA 3.5. Let $\mathcal{P}$ be a set of $m$ polygons, where every pair of them intersects at most $\zeta$ times. Let $\mathcal{R}_{L}$ and $\mathcal{R}_{T}$ be the sets of all loose and tight corridors, respectively, induced by any subset of polygons of $\mathcal{P}$. We have the following:

(A) A loose corridor has a defining set of size $\leq 4$, and $\left|\mathcal{R}_{L}\right|=O\left(\mathrm{~m}^{4}\right)$.

(B) A tight corridor has a defining set of size $\leq 12$, and $\left|\mathcal{R}_{T}\right|=O\left(\mathrm{~m}^{12} \zeta^{8}\right)$.

Proof. It is easy to verify that a loose polygon is defined by at most four polygons-indeed, two polygons define the floor and ceiling chains, and two additional polygons define the start and end vertices. In particular, $\left|\mathcal{R}_{L}\right|=O\left(m^{4}\right)$.

A tight corridor is defined by vertices and subcurves of $\mathcal{A}(\mathcal{P})$. As such, to bound the number of tight corridors, we first bound the number of such entities in the arrangement $\mathcal{A}(\mathcal{P})$. A vertex of $\mathcal{A}(\mathcal{P})$ is the intersection point (of the boundary) of two polygons $\sigma, \tau \in \mathcal{P}$. Since there are $\leq \zeta$ intersection points between $\partial \sigma$ and $\partial \tau$, it follows that a vertex can be specified uniquely by these two polygons and an integer in $\llbracket \zeta \rrbracket$. Thus, overall, there are $\left(\begin{array}{c}m \\ 2\end{array}\right) \zeta$ vertices in $\mathcal{A}(\mathcal{P})$.

A subcurve of $\mathcal{A}(\mathcal{P})$ starts at vertex $u$, that is, induced by two polygons $\sigma, \tau \in \mathcal{P}$, and follows (say) $\tau$, until it reaches a new vertex $v$ that is the intersection of $\partial \tau$ with the boundary of some polygon $\phi \in \mathcal{P}$. As such, the number of such subcurves in $\mathcal{A}(\mathcal{P})$ is $\leq m^{3} \zeta^{2}$, and such a subcurve is defined by three input polygons.

Now, as in the loose case, a tight corridor is defined by four entities-in the loose case, these were four polygons, while in the tight case these are subcurves. It follows that a tight corridor is defined by at most twelve polygons of $\mathcal{P}$, and the number of such corridors is at most $\left(m^{3} \zeta^{2}\right)^{4}=m^{12} \zeta^{8}$.

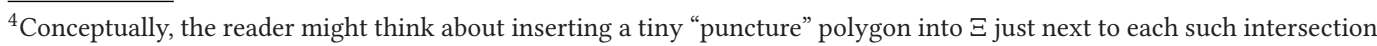
vertex, and applying Lemma 3.3 to this set of polygons, where each connected component of the boundary is a polygon.
} 
LEMma 3.6. Let $\mathcal{P}$ be a set of polygons, such that the boundary of any pair of them intersects at most $\zeta$ times, and let $\mathcal{S} \subseteq \mathcal{P}$ be a set of $m$ polygons. Then, the number of corridors in $\mathcal{C}(\mathcal{S})$ (i.e., the complexity of $\mathcal{C}(\mathcal{S}))$ is $u(m)=O(m)$ if the polygons of $\mathcal{S}$ are disjoint, and $u(m)=O\left(\zeta m^{2}\right)$ otherwise.

Proof. The disjoint case is immediate from Lemma 3.3. As for the more general case, observe that the arrangement $\mathcal{A}(\mathcal{S})$ has at most $\zeta\left(\begin{array}{c}m \\ 2\end{array}\right)$ intersection vertices. The bound now follows by applying Lemma 3.4 .

\subsection{Sampling, Exponential Decay, and Cuttings}

We next show how compute $1 / \boldsymbol{r}$-cuttings for a collection of disjoint polygons, and for sparse sets of polygons. We start by reproving the exponential decay lemma.

3.2.1 Exponential Decay. Let $\mathcal{P}=\left\{\sigma_{1}, \ldots, \sigma_{m}\right\}$ be a set of $m$ polygons in the plane, where every polygon $\sigma_{i} \in \mathcal{P}$ has assigned weight $w_{i}>0$, and let $W=\sum_{i=1}^{m} w_{i}$. We consider two cases: when $\mathcal{P}$ is independent, and when the polygons in $\mathcal{P}$ can intersect.

Consider any subset $\mathcal{S} \subseteq \mathcal{P}$. It is easy to verify that the following two conditions hold.

(i) For any $C \in \mathcal{C}(\mathcal{S})$, we have $D(C) \subseteq \mathcal{S}$ and $\mathcal{S} \cap K(C)=\emptyset$, where $D(C)$ and $K(C)$ are the defining set and the conflict list of $C$, respectively.

(ii) If $D(C) \subseteq \mathcal{S}$ and $K(C) \cap \mathcal{S}=\emptyset$, then $C \in \mathcal{C}(\mathcal{S})$.

Namely, the corridor decomposition complies with the technique of Clarkson-Shor [21] (see also Reference [31, Chapter 8]).

Definition 3.7. For a set $\mathcal{P}$ of weighted polygons and a target size $\rho>0$, a $\boldsymbol{\rho}$-sample is a random sample $\mathcal{S} \subseteq \mathcal{P}$, where each polygon $\sigma_{i} \in \mathcal{P}$ is in $\mathcal{S}$ with probability $\rho w_{i} / W$.

Definition 3.8. A monotone increasing function $u(\cdot) \geq 0$ is polynomially growing, if for any integer $i>0$ we have that $u(i n) \leq i^{O(1)} u(n)$.

We next prove a weighted version of the exponential decay lemma-this is a standard implication of the Clarkson-Shor technique. The proof is a straightforward extension of the standard proof (if slightly simpler) and is presented here for the sake of completeness.

Lemma 3.9 (Weighted exponential DecAy Lemma). Let $\mathcal{P}=\left\{\sigma_{1}, \ldots, \sigma_{m}\right\}$ be a set of $m$ disjoint polygons in the plane, with weights $w_{1}, \ldots, w_{m}$, respectively. Let $\rho \leq m$ and $1 \leq t \leq \rho / 4$ be parameters, and let $W=\sum_{i} w_{i}$. Consider two independent random $\rho$-samples $\mathcal{S}_{1}$ and $\mathcal{S}_{2}$ of $\mathcal{P}$, and let $\mathcal{S}=\mathcal{S}_{1} \cup \mathcal{S}_{2}$. A corridor $C \in \mathcal{C}(\mathcal{S})$ is $\boldsymbol{t}$-heavy if the total weight of the polygons of $\mathcal{P}$ in its conflict list $K(C)$ is at least $t W / \rho$. Let $C_{\geq t}(\mathcal{S})$ be the set of all theavy corridors of $\mathcal{C}(\mathcal{S})$. We have that $\mathrm{E}\left[\left|C_{\geq t}(\mathcal{S})\right|\right]=O(\rho \exp (-t))$.

In a more general setting, when the polygons in $\mathcal{P}$ are not disjoint, and the corridor decomposition of any $m^{\prime}$ of them has complexity $u\left(m^{\prime}\right)$, where $u\left(m^{\prime}\right)$ is a polynomially growing function, we have that $\mathrm{E}\left[\left|\mathcal{C}_{\geq t}(\mathcal{S})\right|\right]=O(u(\rho) \exp (-t))$.

Proof. The basic argument is to use double sampling. Intuitively (but outrageously wrongly), a heavy corridor of $\mathcal{C}(\mathcal{S})$ has constant probability to be present in $\mathcal{C}\left(\mathcal{S}_{1}\right)$, but then it has exponentially small probability (i.e., $e^{-t}$ ) of not being "killed" by a conflicting polygon present in the second sample $\mathcal{S}_{2}$.

For a polygon $\tau \in \mathcal{P}$, we have that $\operatorname{Pr}\left[\tau \in \mathcal{S}_{1} \mid \tau \in \mathcal{S}\right]=\operatorname{Pr}\left[\tau \in \mathcal{S}_{2} \mid \tau \in \mathcal{S}\right] \geq 1 / 2$. Now, consider a corridor $C \in \mathcal{C}(\mathcal{S})$, and let $\tau_{1}, \ldots, \tau_{b} \in \mathcal{P}$ be its defining set, where $b$ is some small constant. 
Clearly, we have that

$$
\begin{aligned}
\operatorname{Pr} & {\left[C \in \mathcal{C}\left(\mathcal{S}_{1}\right) \mid C \in \mathcal{C}(\mathcal{S})\right]=\operatorname{Pr}\left[\tau_{1}, \ldots, \tau_{b} \in \mathcal{S}_{1} \mid C \in \mathcal{C}(\mathcal{S})\right] } \\
& =\prod_{i=1}^{b} \operatorname{Pr}\left[\tau_{i} \in \mathcal{S}_{1} \mid C \in \mathcal{C}(\mathcal{S})\right]=\prod_{i=1}^{b} \operatorname{Pr}\left[\tau_{i} \in \mathcal{S}_{1} \mid \tau_{1}, \ldots, \tau_{b} \in \mathcal{S}\right] \\
& =\prod_{i=1}^{b} \operatorname{Pr}\left[\tau_{i} \in \mathcal{S}_{1} \mid \tau_{i} \in \mathcal{S}\right] \geq \frac{1}{2^{b}} .
\end{aligned}
$$

This in turn implies that

$$
2^{b} \operatorname{Pr}\left[\left(C \in \mathcal{C}\left(\mathcal{S}_{1}\right)\right) \cap(C \in \mathcal{C}(\mathcal{S}))\right] \geq \operatorname{Pr}[C \in \mathcal{C}(\mathcal{S})] .
$$

Next, consider a corridor $C \in \mathcal{C}\left(\mathcal{S}_{1}\right)$ that is $t$-heavy, with, say, $\left\{\sigma_{1}, \ldots, \sigma_{k}\right\} \subseteq \mathcal{P}$ being its conflict list. Clearly, the probability that $\mathcal{S}_{2}$ fails to pick one of the conflicting polygons in $\mathcal{S}_{2}$, is bounded by

$$
\begin{aligned}
\operatorname{Pr}\left[C \in \mathcal{C}(\mathcal{S}) \mid C \in \mathcal{C}\left(\mathcal{S}_{1}\right)\right] & =\operatorname{Pr}\left[\forall i \in\{1, \ldots, k\} \quad \sigma_{i} \notin \mathcal{S}_{2}\right]=\prod_{i=1}^{k}\left(1-\rho \frac{w_{i}}{W}\right) \\
& \leq \prod_{i=1}^{k} \exp \left(-\rho \frac{w_{i}}{W}\right)=\exp \left(-\frac{\rho}{W} \sum_{i=1}^{k} w_{i}\right) \\
& \leq \exp \left(-\frac{\rho}{W} \cdot t \frac{W}{\rho}\right)=e^{-t} .
\end{aligned}
$$

Let $\mathcal{F}$ be the set of possible corridors that can be present in the corridor decomposition of any subset of polygons from $\mathcal{P}$, and let $\mathcal{F}_{\geq t} \subseteq \mathcal{F}$ be the set of all $t$-heavy corridors from $\mathcal{F}$. We have that

$$
\begin{aligned}
\mathrm{E}\left[\left|\mathcal{C}_{\geq t}(\mathcal{S})\right|\right] & =\sum_{C \in \mathcal{F}_{\geq t}} \operatorname{Pr}[C \in \mathcal{C}(\mathcal{S})] \leq \sum_{C \in \mathcal{F}_{\geq t}} 2^{b} \operatorname{Pr}\left[\left(C \in \mathcal{C}\left(\mathcal{S}_{1}\right)\right) \cap(C \in \mathcal{C}(\mathcal{S}))\right] \\
& \leq 2^{b} \sum_{C \in \mathcal{F}_{\geq t}} \underbrace{\operatorname{Pr}\left[C \in \mathcal{C}(\mathcal{S}) \mid C \in \mathcal{C}\left(\mathcal{S}_{1}\right)\right]}_{\leq e^{-t}} \operatorname{Pr}\left[C \in \mathcal{C}\left(\mathcal{S}_{1}\right)\right] \leq 2^{b} e^{-t} \sum_{C \in \mathcal{F}_{\geq t}} \operatorname{Pr}\left[C \in \mathcal{C}\left(\mathcal{S}_{1}\right)\right] \\
& \leq 2^{b} e^{-t} \sum_{C \in \mathcal{F}} \operatorname{Pr}\left[C \in \mathcal{C}\left(\mathcal{S}_{1}\right)\right]=2^{b} e^{-t} \mathrm{E}\left[\left|\mathcal{C}\left(\mathcal{S}_{1}\right)\right|\right] .
\end{aligned}
$$

If $\mathcal{P}$ is a set of disjoint polygons, by Lemma 3.3, and since $\mathbf{E}\left[\left|\mathcal{S}_{1}\right|\right]=\rho$ and $b$ is a constant, then we obtain $\mathrm{E}\left[\left|C_{\geq t}(\mathcal{S})\right|\right]=O\left(e^{-t} \rho\right)$.

As for the second claim, by the Chernoff inequality, and since $u(\cdot)$ is polynomially growing, there are constants $c$ and $c^{\prime}$, such that

$$
\mathbf{E}\left[\left|\mathcal{C}\left(\mathcal{S}_{1}\right)\right|\right] \leq u(\rho)+\sum_{i=1}^{\infty} \operatorname{Pr}\left[\left|\mathcal{S}_{1}\right| \geq i \rho\right] u((i+1) \rho) \leq u(\rho)+\sum_{i=1}^{\infty} 2^{-i} c(i+1)^{c^{\prime}} u(\rho)=O(u(\rho)) .
$$

We obtain $\mathrm{E}\left[\left|C_{\geq t}(\mathcal{S})\right|\right]=O\left(e^{-t} u(\rho)\right)$.

Our proof of the exponential decay lemma is inspired by the work of Sharir [49]. The resulting computations seem somewhat easier than the standard argumentation.

Remark 3.10. The proof Lemma 3.9 assumes that each possible corridor has a unique defining set. Under general position assumption this is a reasonable assumption. However, if this does not hold, then it is not too difficult to resolve this issue-one treats every corridor as labeled by its 
defining set-thus creating several copies of the same corridor. We then give priority to copies of the same corridor by their lexicographical ordering.

3.2.2 Cuttings. For a set $\mathcal{P}$ of weighted polygons of total weight $W$, and a parameter $r \in \mathbb{N}$, a $1 / r$-cutting is a decomposition $\mathcal{C}$ of the plane into corridors, such that

(A) the total number of regions in $\mathrm{C}$ is small, and

(B) for a $C \in \mathcal{C}$, the total weight of the polygons of $\mathcal{P}$ that their boundary intersects the interior of $C$ is at most $W / r .^{5}$

See References [11, 16, 29] and references therein for more information about cuttings.

In the following, complexity of a corridor decomposition refers to its combinatorial complexity, Intuitively, this is the complexity of describing the corridor decomposition once the input is already provided (as a vertex might be the intersection of two input entities, etc.). For more details about combinatorial complexity of arrangements, see the book by Sharir and Agarwal [48].

LEMMA 3.11. Let $\mathcal{P}$ be a set of weighted polygons of total weight $W$, not necessarily disjoint, such that for any subset $\mathcal{S} \subseteq \mathcal{P}$, the complexity of $\mathrm{C}(\mathcal{S})$ is $u(|\mathcal{S}|)$, and $u(\cdot)$ is a polynomially growing function. Then for any parameter $r \in \mathbb{N}$ there exists a $1 / r$-cutting for $\mathcal{P}$, which consists of $O(u(\rho))$ corridors, where $\rho=O(r \log r)+r \ln u(2)$. Furthermore, this cutting can be computed efficiently.

Proof. Let $\mathcal{S}_{1}$ and $\mathcal{S}_{2}$ be two independent random $\rho$-samples of $\mathcal{P}$ for $\rho=r(c \ln r+\ln u(2))$, where $c$ is a sufficiently large constant, and let $\mathcal{S}=\mathcal{S}_{1} \cup \mathcal{S}_{2}$. We claim that the corridor decomposition $\mathcal{C}(\mathcal{S})$ is the desired cutting.

Since $u(\cdot)$ is polynomially growing, it must be that $u(i) \leq u(2) i^{O(1)}$. Now, a corridor $C \in \mathcal{C}(\mathcal{S})$ such that the polygons on the conflict list of $C$ have the total weight of at least $W / r$ is $t$-heavy for $t=c \ln r+\ln u(2)$. By Lemma 3.9, the number of such corridors is in expectation

$$
\mathrm{E}\left[\left|C_{\geq t}(\mathcal{S})\right|\right]=O(u(\rho) \exp (-t))=O\left(u(2) \rho^{O(1)} \exp (-t)\right)=O\left(u(2) r^{O(1)} \exp (-t)\right)<\frac{1}{r^{O(1)}},
$$

for a sufficiently large constant $c$. By Markov's inequality, we have $\operatorname{Pr}\left[\left|C_{\geq t}(\mathcal{S})\right| \geq 1\right] \leq$ $\mathrm{E}\left[\left|C_{\geq t}(\mathcal{S})\right|\right] \leq 1 / r^{O(1)}$. Namely, with probability $\geq 1-1 / r^{O(1)}$, there are no $t$-heavy corridors in $\mathcal{C}(\mathcal{S})$ - that is, all the corridors of $\mathcal{C}(\mathcal{S})$ have conflict lists with weights $\leq W / r$, as desired.

The expected of size of the decomposition $\mathcal{C}(\mathcal{S})$ is $O(u(\rho))$, as follows from the argument used in Lemma 3.9.

Thus, the $\mathcal{C}(\mathcal{S})$ is a $1 / r$-cutting with probability $\geq 1-1 / r^{O(1)}$, and its (expected) size is $O(u(\rho))$.

Note that one key property of the above lemma is the bound on the number of regions. Our lemma above yields a weaker bound on this than what is known for similar settings in the literature. However, it will be sufficient for our purposes. Note that for disjoint polygons we have that $u(\cdot)$ is linear (see Lemma 3.3), and therefore the cutting has size $O(r \log r)$.

3.2.3 Smaller Cuttings. Getting $1 / r$-cuttings of size $O(u(r))$ (and thus of size $O(r)$ for disjoint polygons) is somewhat more challenging. However, for our purposes, any $1 / r$-cutting of size $O\left(r^{c}\right)$, where $c<2$ is a constant, is sufficient (as provided by Lemma 3.11). Nevertheless, one way to get the smaller cuttings is by restricting the kind of polygons under consideration. We do not use the following lemma in our algorithms but it might be useful for further work.

\footnotetext{
${ }^{5}$ Note that this definition does not bound the total weight of the polygons that fully contain a region of the cutting. Indeed, this quantity can be arbitrarily large.
} 
LEMMA 3.12. Let $\mathcal{P}$ be a set of weighted polygons of total weight $W$, not necessarily disjoint, such that for any subset $\mathcal{S} \subseteq \mathcal{P}$, the complexity of $\mathrm{C}(\mathcal{S})$ is $u(|\mathcal{S}|)$, and $u(\cdot)$ is a polynomially growing function. In addition, assume that every polygon in $\mathcal{P}$ has $O(1)$ intersection points with any line, and the boundaries of every pair of polygons of $\mathcal{P}$ have a constant number of intersections. Then for any parameter $r \in \mathbb{N}$ there exists a $1 / r$-cutting of $\mathcal{P}$, which consists of $O(u(r))$ regions, where every region is the intersection of two corridors. Furthermore, this cutting can be computed efficiently.

Proof SKETCH. In this case, $u(2)=O(1)$, since a pair of polygons intersect only a constant number of times. As such, the result follows by the standard two level sampling used in the regular cutting construction. Specifically, we first take a corridor decomposition $\mathcal{C}(\mathcal{S})$ corresponding to a sample $\mathcal{S}$ of size $r$. Then we fix any corridor $C \in \mathcal{C}(\mathcal{S})$ such that the polygons in the conflict list of $C$ are too heavy, by doing a second level sampling. We are using Lemma 3.11 here. In the resulting decomposition, we have to clip every corridor generated in the second level, to its parent corridor. The assumption about every polygon intersecting any line at most some constant number of times implies the desired bound. We omit any further details-see de Berg and Schwarzkopf [11] and Chazelle and Friedman [16].

\subsection{Structural Lemma about a Good Separating Polygon}

Lemma 3.13. Consider a set $\mathcal{P}$ of $m$ weighted polygons of total complexityn, not necessarily disjoint. Let $O$ be a maximum weight independent set of polygons in $\mathcal{P}$, where $W_{\mathrm{opt}}:=w(O)=\sum_{\sigma \in O} w(\sigma)$. Let $r$ be a parameter. Then there exists a polygon $\Gamma$ satisfying the following conditions.

(A) The total weight of the polygons of $O$ completely inside (respectively, outside of) $\Gamma$ is at least $\frac{1}{10} W_{\text {opt }}$.

(B) The total weight of the polygons of $O$ that intersect the boundary of $\Gamma$ is $O\left(\sqrt{\frac{\log r}{r}} W_{\text {opt }}\right)$.

(C) The polygon $\Gamma$ can be fully encoded by a binary string of $O(\sqrt{r \log r} \log m)$ bits.

Proof. Let $\mathcal{C}$ be a $1 / r$-cutting of $O$, as computed by the algorithm of Lemma 3.11. Here, since $O$ is a set of disjoint polygons, the complexity of the corridor decomposition of any subset of $v$ of them is $u(v)=O(v)$, by Lemma 3.6. As such, $\mathcal{C}$ is a decomposition of the plane into $\rho=O(r \log r)$ corridors (there are also the polygons that define the cutting $\mathcal{C}$-we treat them too as corridors that are part of the decomposition $\mathcal{C}$ ).

We interpret $\mathcal{C}$ as a planar map with $O(\rho)$ faces, and assign every polygon $\sigma \in O$ to the corridor $C \in \mathcal{C}$, which contains the leftmost vertex of $\sigma$. As such, the weight of a corridor $C \in \mathcal{C}$ is the total weight of the polygons that have been assigned to $C$. Notice that although a polygon of $O$ might intersect several corridors, it is assigned to only one of them.

Now consider the dual graph $\mathrm{D}$, where every corridor $C \in \mathcal{C}$ corresponds to a vertex in $\mathrm{D}$, and two vertices are connected by an edge if the corresponding corridors are adjacent, see Figure 4(A). The dual graph is connected, but potentially it might contain self-loops, parallel edges, and vertices of degree one. We now fix the dual graph so that it becomes triangulated and has none of these "bad" features. To this end, we replace some of the vertices of D by a set of vertices, as follows.

(A) We replace each vertex $u$ of degree one by two vertices $u_{1}$ and $u_{2}$, and the only edge $u v$ adjacent to $u$ by two edges $u_{1} v$ and $u_{2} v$. We also introduce an edge $u_{1} u_{2}$ between the two new vertices. We do a similar reduction if the vertex is of degree two. After this operation all the vertices of $D$ have a degree of at least three.

(B) If a vertex $u$ of degree $d$ has self loops or parallel edges, then we replace it by $d$ new vertices $u_{1}, \ldots, u_{d}$ that are connected in a cycle. We triangulate the inner cycle, and redirect the $i$ th edge of $u$ to $u_{i}$. 


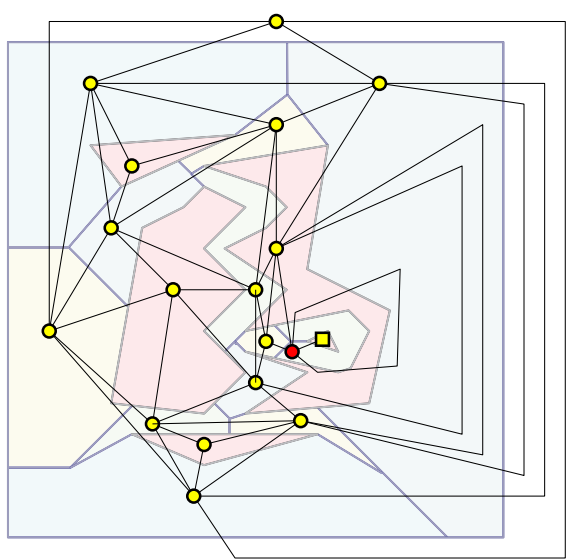

(A) The corridor decomposition (see Figure 2), and its dual graph.

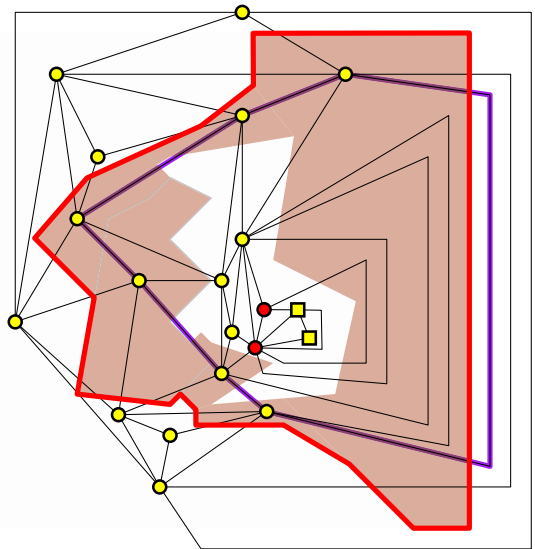

(C) A separating cycle, and the outer boundary of the union of corresponding corridors, which is the separating polygon $\Gamma$.

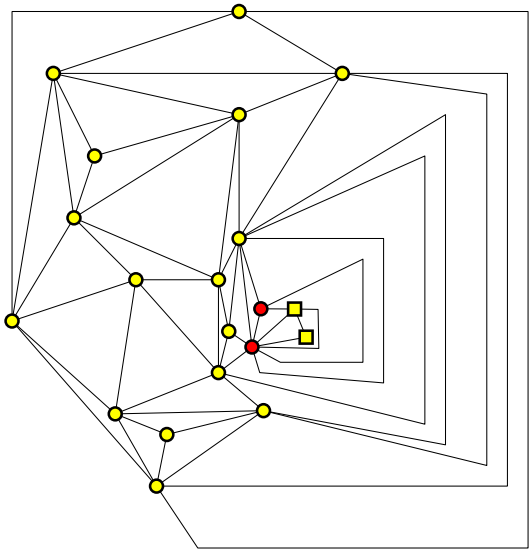

(B) Fixed and triangulated dual graph.

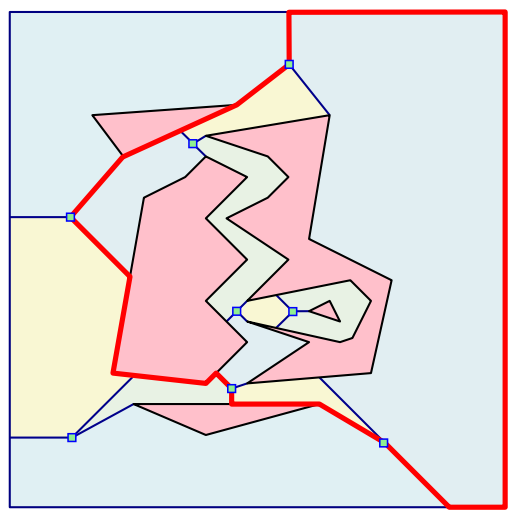

(D) The separating polygon $\Gamma$ in the original corridor decomposition.

Fig. 4. Computing a balanced separator from a cutting.

Finally, we triangulate the resulting graph (i.e., we add edges, that are not necessarily straight segments, until every face has three edges on its boundary), and let $\mathrm{D}^{\prime}$ be the resulting graph, see Figure 4(B). Every vertex $v \in \mathrm{V}(\mathrm{D})$ is associated with a set of vertices $D(v)$ in $\mathrm{D}^{\prime}$. We assign the weight of $v$ arbitrarily to one of the vertices of $D(v)$, and all the other vertices of $D(v)$ are assigned weight 0 . It is easy to verify that $\left|\mathrm{V}\left(\mathrm{D}^{\prime}\right)\right|=O(|\mathrm{~V}(\mathrm{D})|)$.

Now $D^{\prime}$ has a cycle separator $\Gamma^{\prime}$ such that the total weight of the vertices inside (outside, respectively) the cycle $\Gamma^{\prime}$ is at most $(3 / 4) W_{\text {opt }}$, and $\Gamma^{\prime}$ has at most $4 \sqrt{\left|\mathrm{V}\left(\mathrm{D}^{\prime}\right)\right|}$ vertices-this follows from the cycle separator of Miller [43] (which is weighted). The resulting cycle visits $M=O(\sqrt{\rho})$ vertices of $\mathrm{D}^{\prime}$, which corresponds to a set $\mathrm{C}^{\prime}$ of at most $M$ corridors of $\mathcal{C}$. One can now track a closed curve $\Gamma^{\prime \prime}$ in the plane, corresponding to the cycle $\Gamma^{\prime}$ in the primal, so that the curve stays inside the union of the corridors of $\mathrm{C}^{\prime}$, and all the vertices of $\mathrm{D}^{\prime}$ inside (respectively, outside of) $\Gamma^{\prime}$ correspond to the corridors that are strictly inside (respectively, outside of) the curve $\Gamma^{\prime \prime}$. Now, $\Gamma^{\prime \prime}$ can be transformed to a curve $\Gamma$ using only the boundary of the corridors of $\mathcal{C}^{\prime}$-the easiest 
way to do so, is to take $\Gamma$ to be the outer boundary of the union of all the corridors of $\mathrm{C}^{\prime}$, see Figure 4(D). As such, $\Gamma$ consists of $O(\sqrt{\rho})$ edges. Here, an edge is either a spoke or a subchain of one of the polygons of $\mathcal{P}$. Now, the total weight of polygons of $\mathcal{P}$ that intersect a spoke ${ }^{6}$ used in the $1 / r$-cutting can be at most $W_{\text {opt }} / r$, it follows that the total weight of polygons in $O$ intersecting $\Gamma$ is

$$
O\left(\sqrt{\rho} \frac{W_{\mathrm{opt}}}{r}\right)=O\left(\sqrt{r \log r} \cdot \frac{W_{\mathrm{opt}}}{r}\right)=O\left(W_{\mathrm{opt}} \sqrt{\frac{\log r}{r}}\right) .
$$

We next show how to encode each edge of $\Gamma$ using $O(\log m)$ bits, which implies the claim. Compute the set of $O\left(\mathrm{~m}^{4}\right)$ loose corridors induced by any subset of polygons of $\mathcal{P}$ that do not intersect, see Lemma 3.5. Let $\mathcal{X}$ be this set of polygons. Every corridor of $\mathcal{X}$ induces $\leq 4$ vertices where its spokes touch its two adjacent polygons. In particular, let $Q$ be the set of all such vertices. Clearly, there are $O\left(\mathrm{~m}^{4}\right)$ such vertices.

Consider an edge $e$ of $\Gamma$. If it is a spoke, then we can encode it by specifying which spoke it is, which requires $O\left(\log m^{4}\right)$ bits, since there are $O\left(m^{4}\right)$ possible spokes. Otherwise, the edge is a subchain of one of the polygons of $\mathcal{P}$. We specify which one of the polygons it is on, which requires $O(\log m)$ bits, and then we specify that start and end vertices, which belong to $\mathrm{Q}$, which requires $O(\log m)$ bits. We also need to specify which one of the two possible polygonal subchains we refer to, which requires an extra bit. Overall, the number of bits needed to encode $\Gamma$ is $O(\sqrt{\rho} \log m)$, as claimed.

Remark 3.14. If one polygon in the optimal solution is heavier than a $W_{\text {opt }} / 10$, then the cut can be the polygon itself-the polygon defines the inner subproblem, and all polygons that do not intersect it are the outside subproblem. This degenerate case is implicit in Lemma 3.13, and is not described explicitly, for the sake of simplicity.

Remark 3.15. The proof of Lemma 2.3 follows from Lemma 3.13 by choosing $r:=\left(\frac{\log m}{\varepsilon}\right)^{2+\mu}$ for any $\mu>0$.

Remark 3.16. The separating cycle of Lemma 3.13 is defined by a random sample of the optimal solution. An interesting property of the construction is that these defining polygons are added as their own corridors to the constructed arrangement. These defining polygons are "islands" in the constructed arrangement, and the corridor decomposition tiles their complement. It thus follows that the constructed separating cycle does not intersect the interior of the defining polygons. This is crucial, as if there are a few heavy polygons (say, of weight $\geq \varepsilon W_{\text {opt }}$ ), then they would be part of the defining set of the cycle, and they would get sent down to one of the two recursive subproblems.

Remark 3.17. While the separating polygon $\Gamma$ has a short encoding, it potentially can have a large number of edges $-O(n)$ in the worst case, where $n$ is the total number of vertices in the input polygon. The separating polygon $\Gamma$ is a simple polygon (i.e., no holes or self intersections). Thus, $\Gamma$ can be preprocessed in $O(n \log n)$ time for ray shooting (from the inside and outside), where a ray shooting query can be answered in $O(\log n)$ time [35]. Now, given another polygon $\sigma$, one can decide if $\sigma$ is intersecting, inside or outside of $\Gamma$ by checking for each edge of $\sigma$ whether it intersects the boundary of $\Gamma$, and deciding (say using a point-location data-structure) if a vertex of $\sigma$ is inside $\Gamma$. As such, given a set of polygons with a total of $n$ vertices, one can partition them into the inside/outside/intersecting sets, in relation to $\Gamma$, in $O(n \log n)$ time.

\footnotetext{
${ }^{6}$ Note that by the disjointness of the polygons of $O$, no such polygonal chain can intersect any of the polygons of $O$.
} 


\subsection{Extension: QPTAS for Sparse Properties}

When we compute an independent set of polygons, we output a collection of polygons with the property that their intersection graph consists of only isolated vertices. In this section we extend our reasoning to the setting where the output polygons may overlap, but where we require that the intersection graph of the output polygons fulfills some given sparsity condition, i.e., it is planar or it does not contain a $K_{s, t}$ subgraph for some constants $s, t$.

Let $\mathcal{P}$ be a set of polygons in the plane such that no input polygon is contained in another input polygon. We are interested in the intersection graph $\mathrm{G}=(\mathcal{P}, \mathrm{E})$ induced by $\mathcal{P}$; that is, $\mathrm{E}=$ $\{\sigma \tau \mid \sigma, \tau \in \mathcal{P}, \sigma \cap \tau \neq \emptyset\}$. For a subset $X \subseteq \mathcal{P}$, let $\mathrm{G}_{X}=\left(X, \mathrm{E}_{X}\right)$ denote the induced subgraph of $\mathrm{G}$ on $X$; that is, $\mathrm{E}_{X}=\{\sigma \tau \mid \sigma, \tau \in X$ and $\sigma \tau \in \mathrm{E}\}$. We refer to two subsets $X \subseteq \mathcal{P}$ and $Y \subseteq \mathcal{P}$ as separate, if no polygon of $X$ intersects any polygon in $Y$.

Consider a property $\Pi$ on graphs (e.g., a graph is planar). We can naturally define the set system of all subsets of $\mathcal{P}$ that have this property. That is $\Pi_{\mathcal{P}}=(\mathcal{P}, \mathcal{I})$, where $\mathcal{I}=$ $\left\{X \subseteq \mathcal{P} \mid \mathrm{G}_{X}\right.$ has property $\left.\Pi\right\}$.

We are interested here in hereditary properties. Specifically, if $X \in \Pi_{\mathcal{P}}$, then $Y \in \Pi_{\mathcal{P}}$, for all $Y \subseteq X$. We also require that the property would be mergeable; that is, for any two separate subsets $X, Y \subseteq \mathcal{P}$, such that $X, Y \in \Pi_{\mathcal{P}}$ we have that $X \cup Y \in \Pi_{\mathcal{P}}$. Notice that the combinatorial structure $\Pi_{\mathcal{P}}$ is similar to a matroid, except that we do not require to have the augmentation property (this is also known as an independence system).

Here, unlike the independent set case, we assume that the input polygons are unweighted, see Remark 3.22 below for more details. The purpose here is to compute (or approximate) the maximum cardinality set $X \in \Pi_{\mathcal{p}}$.

As a concrete example, consider the property $\Pi$ that a set $X \subseteq \mathcal{P}$ has no pair of intersecting polygons. In this case, finding the maximum cardinality set in $\Pi_{\mathcal{P}}$ that has the desired property corresponds to finding the maximum independent set in $\mathcal{P}$.

Definition 3.18. A property $\Pi_{\mathcal{P}}$ is sparse if there are constants $\delta, c>0$, such that for any $X \in$ $\Pi_{\mathcal{P}}$, we have that $\left|\mathrm{E}\left(\mathrm{G}_{X}\right)\right| \leq c|X|^{2-\delta}$.

Informally, sparsity implies that in any set $X \in \Pi_{\mathcal{P}}$ the number of pairs of intersecting polygons is strictly subquadratic in the size of $X$. Surprisingly, for an intersection graph of curves where every pair of curves intersects only a constant number of times, sparsity implies that the number of edges in the intersection graph is linear [26].

Lemma 3.19 ([26]). Let $\mathcal{P}$ be a set of polygons such that the boundaries of every pair of polygons have a constant number of intersections, and such that no polygon is contained in another polygon. Let $\Pi_{\mathcal{P}}$ be a sparse property. Then, for any $X \in \Pi_{\mathcal{P}}$, we have that $\left|\mathrm{E}\left(\mathrm{G}_{X}\right)\right|=O(|X|)$.

If a pair of polygons in $\mathcal{P}$ can have $\zeta$ intersections (i.e., of their boundaries), then $\left|\mathrm{E}\left(\mathrm{G}_{X}\right)\right|=$ $O\left(\zeta^{1 / 2}|X|\right)$.

Proof. This result is known [26]. We include a sketch of the proof here for the sake of completeness.

We think about the boundaries of the polygons of $\mathcal{P}$ as curves in the plane, and let $m=|X|$. The intersection graph $G_{X}$ has a subquadratic number of edges, and as such, the arrangement of the curves of $X$ has at most $m^{\prime}=O\left(m^{2-\delta}\right)$ vertices (there is a vertex for each intersection of two curves). By the planar separator theorem, there is a set of $O\left(\sqrt{m^{\prime}}\right)=O\left(m^{1-\delta / 2}\right)$ vertices, that their removal disconnects this arrangement into a set of $m_{1}, m_{2}$ curves, where $m_{1}, m_{2} \leq(2 / 3) m$ and $m_{1}+m_{2} \leq m+O\left(\sqrt{m^{\prime}}\right)$ (here we break the curves passing through a vertex of the separator into two curves, sent to the respective subproblems). Applying the argument now to both sets 
recursively, we get that the total number of vertices is $T(m)=O\left(m^{1-\delta / 2}\right)+T\left(m_{1}\right)+T\left(m_{2}\right)$, and the solution of this recurrence is $T(m)=O(m)$.

If there are $\zeta$ intersections between pairs of polygons, then the associated arrangement has $O\left(\zeta n^{2-\delta}\right)$ vertices, and the recursion becomes $T(m)=O\left(\zeta^{1 / 2} m^{1-\delta / 2}\right)+T\left(m_{1}\right)+T\left(m_{2}\right)$, and the solution is $T(m)=O\left(\zeta^{1 / 2} m\right)$.

A property $\Pi_{\mathcal{P}}$ is exponential time checkable if, for any subset $X \subseteq \mathrm{V}$, one can decide if $X \in$ $\Pi_{\mathcal{P}}$ in time $2^{|X|^{O(1)}}$.

Theorem 3.20. Let $\mathcal{P}$ be a set of $m$ unweighted polygons in the plane, with total of $n$ vertices, such that no input polygon is contained in another input polygon, and such that the boundaries of every pair of them intersect only a constant number of times. Let $\Pi_{\mathcal{P}}$ be a hereditary, sparse and mergeable property that is exponential time checkable. Then, for a parameter $\varepsilon>0$, one can compute in quasi-polynomial time (i.e., $\left.2^{O(\operatorname{poly}(\log m, 1 / \varepsilon))} n^{O(1)}\right)$ a subset $X \subseteq \mathcal{P}$, such that $X \in \Pi_{\mathcal{P}}$, and $|X| \geq$ $(1-\varepsilon)|O|$, where $O$ is the largest set in $\Pi_{\mathcal{P}}$.

Proof. One need to verify that the algorithm of Section 2 works also in this case. As before, we are going to argue that there exists a cheap separating polygon.

So, let $O$ be a maximum weight set in $\Pi_{\mathcal{P}}$, and consider any subset $\mathcal{S} \subseteq O$. Since $\Pi_{\mathcal{P}}$ is hereditary, we have that $\mathcal{S} \in \Pi_{\mathcal{P}}$. By Lemma 3.19 the arrangement $\mathcal{A}(\mathcal{S})$ has $O(|\mathcal{S}|)$ intersection vertices. By Lemma 3.4, the corridor decomposition $\mathrm{C}(\mathcal{S})$ consists of $O(|\mathcal{S}|)$ corridors.

The existence of a good cycle separator now follows from the proof of Lemma 3.13-here the polygons are not necessarily disjoint, but since the corridor decomposition in this case still has linear complexity, it still works.

The resulting separating polygon intersects

$$
O\left(\frac{|O|}{r} \sqrt{r \log r}\right)=O\left(\frac{|O|}{r^{1 / 3}}\right) \leq \frac{\varepsilon}{c \log m}|O|
$$

polygons for $r=\Omega\left((\log m / \varepsilon)^{3}\right)$. It is easy to verify that such a polygon can be encoded using $O($ poly $(\log m, 1 / \varepsilon))$ bits (vertices used by the cycle are either vertices rising from intersection of polygons, and there are $O\left(\mathrm{~m}^{2}\right)$ such vertices, or one of the other $O\left(\mathrm{~m}^{4}\right)$ vertices). The rest of the algorithm now works as described in Section 2. Note that because of the mergeablity assumption the algorithm needs to verify that the generated sets have the desired property only in the bottom of the recursions. But such subsets have size $O(\operatorname{poly}(\log m, 1 / \varepsilon))$, and thus they can be checked in

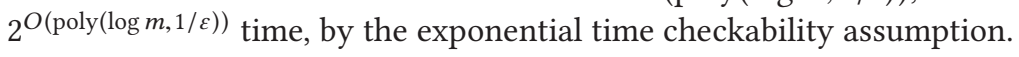

Remark 3.21. If a pair of polygons is allowed to intersect $\zeta$ times (and this number is no longer a constant), then encoding the separating cycle now requires $O\left(\log \left(m^{12} \zeta^{8}\right)\right)$ bits per edge, see Lemma 3.5. The separator now has size $O(\sqrt{\zeta r \log r})$ by Lemma 3.19. The resulting algorithm would still be a QPTAS if for example $\zeta=O(\operatorname{polylog} m)$.

There are known separator results for string graphs where the number of pairwise intersections is not necessarily a constant [42]. Unfortunately, these separators do not necessarily form a cycle, which is crucial for the algorithm to work.

Note that without the assumption that no pair of input polygons is contained inside each other, we have to deal with the non-trivial technicality that the separating cycle might be fully contained inside some input polygon. ${ }^{7}$

\footnotetext{
${ }^{7}$ It seems that this technicality can be handled with some additional care, but the added complexity does not seem to be worth it.
} 
Properties that comply with our conditions, and thus one can now use Theorem 3.20 to get a QPTAS for the largest subset $O$ of $\mathcal{P}$ that have this property, include the following:

(A) All the polygons of $O$ are independent.

(B) The intersection graph of $O$ is planar, or has low genus.

(C) The intersection graph of $O$ does not contain $K_{s, t}$ as a subgraph, for $s$ and $t$ constants.

(D) If the boundaries of every pair of polygons of $\mathcal{P}$ intersects at most twice, then they behave like pseudo-disks. In particular, the union complexity of $m$ pseudo-disks is linear, and the by the Clarkson-Shor technique, the complexity of the arrangement of depth $k$ of $m$ pseudo-disks is $O(\mathrm{~km})$. This implies that if $O$ is a set pseudo-disks with bounded depth, then the intersection graph has only $O(|O|)$ edges, and as such this is a sparse property, and it follows that one can $(1-\varepsilon)$-approximate (in quasi-polynomial time) the largest subset of pseudo-disks where the maximum depth is bounded. Previously, only a constant approximation was known [23].

Remark 3.22. The algorithm of Theorem 3.20 does not work for weighted polygons. ${ }^{8}$ The main reason is that the separating cycle, when applied to the optimal solution, is defined by a collection of polygons. In the independent set case, these defining polygons were not lost (see Remark 3.16), but this can no longer be guaranteed. Unfortunately, these polygons can be "heavy" and form a crucial subset of the optimal solution. A naive way to try and address this issue is to allow an additional set of "special" polygons sent down to the recursive subproblems as being present in the optimal solution. This requires a modification of the mergeablity property, which works in some cases but fails for others (for example, if the intersection graphs of $X \cup Y, Y \cup Z \subseteq \mathcal{P}$ are planar, then this is not necessarily true for $X \cup Y \cup Z$ ). We leave the extension of the algorithm of Theorem 3.20 as an open problem for further research.

Note that the above is not an issue for the unweighted case-all the polygons intersecting the separating cycle can be thrown away-the number of additional polygons in the set defining the separating cycle is small compared to the optimal solution and has no impact on the approximation quality.

\section{A PTAS FOR $\delta$-LARGE RECTANGLES}

In this section, we present a polynomial time approximation scheme for the maximum weight independent set of $\delta$-large rectangles, i.e., for axis-parallel rectangles that have at least one edge that is long with respect to the corresponding edge of the bounding box.

First, we show that there is a way to partition the plane using a set of at most $\left(\frac{1}{\epsilon \delta}\right)^{O(1)}$ line segments, such that the intersected rectangles have small total weight and each face of the partition is a path or a cycle of "width" at most $\delta N$. Note that the latter bound is strictly smaller than the length of the longer edge of each rectangle. In a sense, this partition sparsely describes the topology of the (large) rectangles while losing only rectangles of negligible weight. Each face of the resulting partition consists of at most $\left(\frac{1}{\epsilon \delta}\right)^{O(1)}$ line segments. We then show that we can solve its corresponding subproblem within a $(1+\varepsilon)$-accuracy by a recursive procedure that splits the the face into smaller and smaller subfaces. Importantly, the complexity of each arising subface is also bounded by $\left(\frac{1}{\epsilon \delta}\right)^{O(1)}$. To achieve polynomial running time, we embed the recursion into a dynamic program and show that the number of subproblems to be considered is polynomially bounded (unlike in the QPTAS above where the number of possible subproblems is larger). Note that this might seem like running the QPTAS from Section 2 with $\alpha=O_{\epsilon}(1)$. However, also for

\footnotetext{
${ }^{8}$ In particular, the conference version of this article [32] incorrectly claimed that the algorithm works in this case.
} 
this choice the resulting running time is only quasi-polynomial and therefore we need a different approach here.

We first present our algorithm for blocks, i.e., for large rectangles whose height or width is 1 , and then we extend it to arbitrary $\delta$-large rectangles.

\subsection{Formal Definition of the Problem}

Let us fix constants $\delta>0$ and $\varepsilon>0$. Let $\mathcal{R}=\left\{\mathrm{r}_{1}, \ldots, \mathrm{r}_{m}\right\}$ be a set of $m$ axis-parallel rectangles with integer coordinates in the plane, where the $i$ th rectangle $r_{i} \in \mathcal{R}$ is defined as an open set $\mathrm{r}_{i}=\left(x_{i}, x_{i}^{\prime}\right) \times\left(y_{i}, y_{i}^{\prime}\right)$, where $x_{i}<x_{i}^{\prime}, y_{i}<y_{i}^{\prime}$. Let $N$ be the smallest integer s.t. the vertices of all rectangles in $\mathcal{R}$ are in $\llbracket N \rrbracket^{2}$. For each rectangle $r_{i}$, its width is $d x_{i}=x_{i}^{\prime}-x_{i}$ and its height is $d y_{i}=y_{i}^{\prime}-y_{i}$. We denote the quantity $\Delta:=\delta N$ as the largeness threshold. A rectangle $r_{i} \in \mathcal{R}$ is $\boldsymbol{\delta}$-large (or just large) if $d y_{i}=y_{i}^{\prime}-y_{i}>\Delta$ or $d x_{i}=x_{i}^{\prime}-x_{i}>\Delta$.

Problem 4.1 (Independent Set of LARge Rectangles). The input consists of a set $\mathcal{R}$ of weighted $\delta$-large rectangles, where the weight of a rectangle $r_{i} \in \mathcal{R}$ is a positive number $w_{i}$. The task is to compute a maximum weight subset $\mathcal{R}^{\prime} \subseteq \mathcal{R}$, such that the rectangles of $\mathcal{R}^{\prime}$ are disjoint.

Definition 4.2. A rectangle $\mathrm{r}_{i} \in \mathcal{R}$ is a block if (i) $\mathrm{r}_{i}$ is $\delta$-large and (ii) either $d y_{i}=1$ or $d x_{i}=1$.

Problem 4.3 (Independent Set of Blocks). The input consists of a set $\mathcal{B}=\left\{\mathrm{b}_{1}, \mathrm{~b}_{2}, \ldots, \mathrm{b}_{m}\right\}$ of weighted blocks. The task is to compute a maximum weight subset $\mathcal{B}^{\prime} \subseteq \mathcal{B}$ such that the rectangles of $\mathcal{B}^{\prime}$ are disjoint.

To simplify the description, we assume the following: (i) $\mathrm{m} / \varepsilon$ and $\mathrm{cm}$ are both integers, (ii) $1 / \delta \in \mathbb{N}$, and (iii) $\Delta=\delta N \in \mathbb{N}$. For any two points $p, \mathrm{p}^{\prime}$, let $\mathrm{p} \mathrm{p}^{\prime}$ denote the closed straight segment from $p$ to $p^{\prime}$. Similarly, let int $\left(p p^{\prime}\right)$ be the open segment $p p^{\prime} \backslash\left\{p, p^{\prime}\right\}$.

\subsection{Constructing the Partition for Blocks}

4.2.1 Overview and Definitions. As before, we assume that the maximum weight subset $O \subseteq \mathcal{B}$ of disjoint blocks is known to us, and we prove that there is a "cheap" partition that enables one to compute a near-optimal independent set of blocks using dynamic programming.

Specifically, we construct a partition of the bounding box $[0, N]^{2}$ using a set of at most $1 /(\varepsilon \delta)^{4}$ rectilinear (i.e., horizontal and vertical) line segments with integer endpoint coordinates, such that the blocks of $O$ intersected by these segments have a small total weight compared with $w(O)$. Furthermore, each face of the partition is either a simple rectilinear polygon, or a rectilinear polygon with a single rectilinear hole. In either case, the polygon would have "width" of at most $\Delta$, which is strictly smaller than the length of any block. In a sense, this partition sparsely describes the topology of the (large) blocks while intersecting blocks of negligible total weight.

From this point on, a segment refers to a horizontal or vertical (closed) line segment that has integer coordinates with endpoints in $\llbracket N \rrbracket^{2}$.

First, we construct a grid $\mathcal{G}$ of large tiles, consisting of $1 / \delta \times 1 / \delta$ uniform grid cells in the input square $[0, N]^{2}$, i.e., for each $i, j \in\{0, \ldots, 1 / \delta-1\}$ there is a grid cell with coordinates $[i \Delta,(i+$ 1) $\Delta] \times[j \Delta,(j+1) \Delta]$. Note that each grid cell is a closed set.

For a set $X \subseteq \mathbb{R}^{2}$, we use $\operatorname{cl}(X)$ to denote the closure of $X$. We remind the reader that blocks (and rectangles) are open sets, and therefore for a block $\mathrm{b} \in \mathcal{B}, \mathrm{cl}(\mathrm{b})$ is the closed version of $\mathrm{b}$. In the sequel we will sometimes refer to the interior of a segment $\mathrm{s}$ by which we mean $\mathrm{s}$ without its two endpoints.

Definition 4.4. A segment (or a line) s cuts a rectangle $r$ if $r \backslash s$ has two connected components. A segment $s$ hits a rectangle $r$ if (i) $s$ intersects $c l(r)$, (ii) $s$ does not intersect $r$, and (iii) line(s) cuts 
$r$, where line(s) denotes the line that spans s. Similarly, a segment s hits a segment t, if (i) s and $t$ are orthogonal to each other and (ii) $s$ has an endpoint in the interior of $t$. Two segments that intersect in their interior are crossing.

Note that if a segment s hits a segment $\mathrm{t}$, then $\mathrm{t}$ does not hit s. By assumption, any block of $\mathcal{B}$ intersects at least two grid cells. A block $\mathrm{b} \in \mathcal{B}$ ends in a grid cell $\square$, if (i) $\square$ and b intersect and (ii) $\square$ contains one of the corners of $b$.

Our construction has two steps. In the first step we construct an initial set of segments $\mathcal{X}$ that contains the boundary of the input square and adds $O(1)$ segments for each grid cell. The segments of $\mathcal{X}$ do not intersect any blocks from the optimal solution $O$ and are pairwise non-crossing, but they might have loose ends, that is, endpoints of segments from $\mathcal{X}$ that are not contained in the interior of some other segment of $\mathcal{X}$. Therefore, in our second step, we add a set of segments $\mathcal{Y}$ that connect these loose ends with other segments in $\mathcal{X} \cup \mathcal{Y}$. Segments in $\mathcal{Y}$ might intersect blocks from $O$; however, the total weight of intersected blocks is bounded by $\varepsilon \cdot w(O)$, and thus we can afford to lose them.

4.2.2 Construction Step I: The Set $\mathcal{X}$. Initially, $\mathcal{X}$ is a set containing the four segments forming the boundary of the input square $[0, N] \times[0, N]$. Next, consider a grid cell $\square$ and its (closed) bottom edge e. A vertical segment $\mathrm{s}$ is admissible if

(i) s intersects e (i.e., either int(s) intersects e, or s has an endpoint on e) and that int(s) intersects $\square$,

(ii) s does not intersect any block of $\mathcal{B}$,

(iii) s does not cross any segment of $\mathcal{X}$,

(iv) $\|s\|>\Delta$ (i.e., $s$ is long), and

(v) $\mathrm{s}$ is a maximal (i.e., as long as possible) segment satisfying the properties above.

We add to $X$ the following segments.

(A) An admissible segment $\mathrm{s}$ with the smallest $x$-coordinate (i.e., s might lie on the left edge of $\square$ )

(B) An admissible segment with the largest $x$-coordinate.

(C) An admissible segment $\mathrm{s}$ that maximizes the length of $\|\mathrm{s} \cap \square\|$. If there are several such segments, then we add two of them: One with the smallest and one with the largest $x$ coordinate, respectively. Segments maximizing $\|s \cap \square\|$ are called reach segments for e in $\square$. The ones added to $\mathcal{X}$ in this step are called extremal reach segments.

The algorithm performs the same operation for the top, left and right edges of $\square$, where for the left and right edges it considers horizontal segments instead of vertical. This is done in a fixed order, e.g., first all vertical segments, and then all horizontal segments. See Figure 5 for an example.

By construction, the resulting segments of $\mathcal{X}$ (excluding the four frame segments) are all interior disjoint and maximal (i.e., they cannot be extended without crossing other segments from $\mathcal{X}$ or intersecting blocks of $O$ ).

\subsubsection{Construction Step II: The Set $\mathcal{Y}$.}

Idea. The segments in $\mathcal{X}$ might have loose ends as mentioned above. We need to connect such endpoints up so that the resulting set of segments partitions the input square into faces, where every face is either a simple polygon, or a polygon with a single hole. The idea is to perform a walk in the square, looking for a way to connect such a loose end with the segments already constructed. If the walk is too long, then it would be shortened by introducing a cheap shortcut segment. 


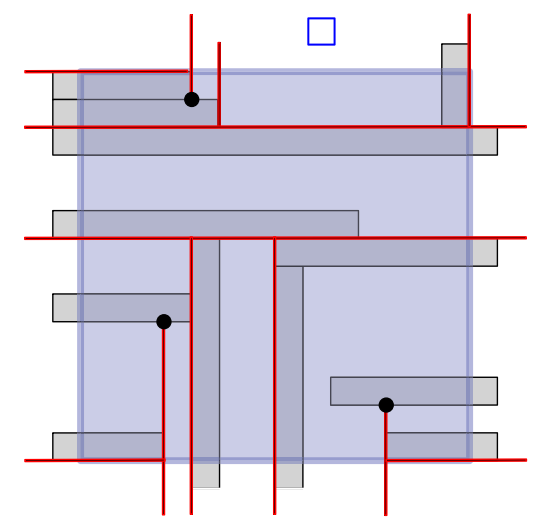

Fig. 5. The red lines denote the segments added to $X$ while processing the grid cell $\square$. The blocks of $O$ intersecting $\square$ are depicted in gray. The black circles denote the loose ends inside $\square$.

Setup. Initially, the set $\mathcal{Y}$ is empty. For each endpoint $\mathrm{p}_{0}$ of a segment $\mathrm{s} \in \mathcal{X}$, such that $\mathrm{s}$ does not hit a perpendicular segment in $\mathcal{X} \cup \mathcal{Y}$ at $\mathrm{p}_{0}$, we create a path of segments connecting $\mathrm{s}$ with a segment in $\mathcal{X} \cup \mathcal{Y}$, adding the new segments of the path to $\mathcal{Y}$.

A segment $s$ is maximal if it does not cross any segment of $\mathcal{X} \cup \mathcal{Y}$ or intersect any of the blocks from $O$, but any segment $s^{\prime}$ such that $s \subset s^{\prime}$ violates this property. Note that any endpoint of a maximal segment must lie in the interior of an edge of a perpendicular block of $O$, or in the interior of a perpendicular segment of $\mathcal{X} \cup \mathcal{Y}$. In the sequel, we use the following technical lemma.

Lemma 4.5. Let $\square$ be a grid cell and $\mathrm{p}$ be a point in $\square$. Let $\mathrm{s}$ be a maximal segment with one endpoint at $\mathrm{p}$, and the other endpoint outside of $\square$. If $\mathrm{s}$ hits a perpendicular block $\mathrm{b} \in O$ at $\mathrm{p}$, but it does not hit a perpendicular segment from $X$ at $\mathrm{p}$, then:

(i) $\mathrm{p} \in \operatorname{int}(\square)$, and

(ii) one end of $\mathrm{b}$ is in $\square$, and the other one is outside of $\square$.

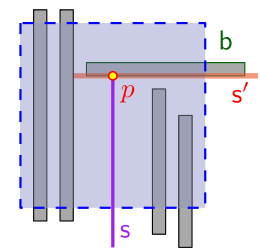

Proof. Assume without loss of generality that $s$ is vertical, $p$ is at the top end of $s$, and $b$ crosses the boundary of the grid cell to the right of $p$ (as, by assumption, $p$ lies on the long edge of $b$ ). Let $s^{\prime}$ be the maximal segment that contains the bottom edge of $b$ and does not intersect any blocks or segments from $\mathcal{X}$. As $p \in \mathrm{s}^{\prime}$, by assumption, we have that $\mathrm{s}^{\prime} \notin \mathcal{X}$.

Assume that $p$ is on the boundary of $\square$. If $p$ lies on the bottom edge of $\square$, then $s^{\prime}$ is the bottommost long segment crossing the right edge of $\square$. But then $\mathrm{s}^{\prime} \in \mathcal{X}$, which is a contradiction. The cases that $p$ is on the top, left or right edges of $\square$ are handled in a similar fashion.

As such, $p \in \operatorname{int}(\square)$ and $b$ intersects the interior of $\square$.

If $b$ does not have an end in $\square$, then s' cuts $\square$. If $\mathrm{s}^{\prime}$ is the bottom-most reach segment for the left edge of $\square$, then $\mathrm{s}^{\prime} \in \mathcal{X}$, which gives a contradiction. Otherwise, the bottom-most reach segment for the left edge of $\square$ is below s' and cuts $\square$, so it intersects s, and again we get a contradiction, as s does not intersect edges from $\mathcal{X}$. Block b must end in $\square$.

Building the path for a single loose end. We consider all loose endpoints of segments in $\mathcal{X}$, one by one. Let $\mathrm{p}_{0}$ be such a loose endpoint of a segment $\mathrm{s}_{0} \in \mathcal{X}$, such that $\mathrm{s}_{0}$ does not hit a perpendicular segment in $\mathcal{X} \cup \mathcal{Y}$ at $\mathrm{p}_{0}$, and set $i=1$. In the following, let

$$
M=128 /\left(\varepsilon \delta^{2}\right) \text {. }
$$

Next, we construct a path $\mathrm{s}_{1}, \ldots, \mathrm{s}_{M}$ starting from $\mathrm{p}_{0}$, aiming to connect $\mathrm{p}_{0}$ with some existing segment in $\mathcal{X} \cup \mathcal{Y}$. 


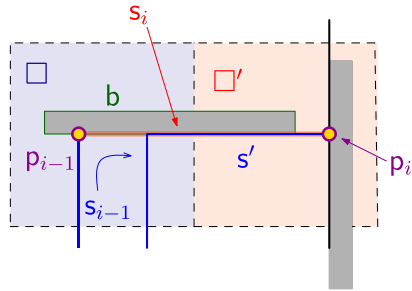

(A)

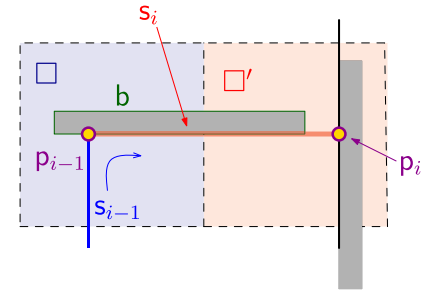

(B)

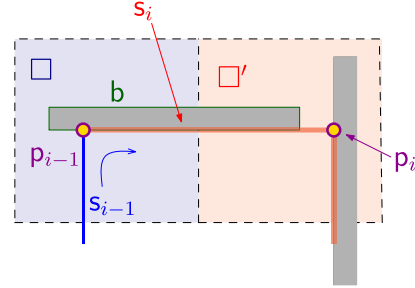

(C)

Fig. 6. Construction of the path. The segment $s_{i-1}$ hits a block $b$ at the point $\mathrm{p}_{i-1}$. The segment $s_{i}$ starts at $\mathrm{p}_{i-1}$ and follows the edge of $\mathrm{b}$ until it hits a perpendicular segment that is already in $\mathcal{X} \cup \mathcal{Y}$ or a perpendicular block. In case $(A)$ the new segment $s_{i}$ overlaps an existing segment $s^{\prime} \in \mathcal{Y} \cup\left\{s_{1}, \ldots, s_{i-1}\right\}$ and $s^{\prime}$ is replaced by $s_{i}$. In case (B), $s_{i}$ is added to $\mathcal{Y}$. In case (C), the walk continues from the new endpoint $\mathrm{p}_{i}$.

Let $\square$ be a grid cell such that $p_{i-1} \in \square$ and $s_{i-1}$ does not hit a segment from $\mathcal{X} \cup \mathcal{Y}$ at $p_{i-1}$. Let $\mathrm{b}$ be the block hit by $\mathrm{s}_{i-1}$ at $\mathrm{p}_{i-1}$. As $\mathrm{s}_{i-1}$ cannot be extended beyond $\mathrm{p}_{i-1}$, such a block $\mathrm{b}$ exists. Applying Lemma 4.5 to the point $\mathrm{p}_{i-1}$ together with the segment $\mathrm{s}_{i-1}$ (we can do that as $s_{i-1}$ intersects at least two cells), we obtain that $p_{i-1} \in \operatorname{int}(\square)$, and $b$ has one end in $\square$ and the other end in some other grid cell $\square^{\prime}$. Let $s_{\max }$ be a maximal segment that contains the edge of $b$ containing $p_{i-1}$. Let $p_{i}$ be the endpoint of $s_{\max }$ such that $\mathrm{p}_{i-1} \mathrm{p}_{i} \cap \square^{\prime} \neq \emptyset$. Set $s_{i}=\mathrm{p}_{i-1} \mathrm{p}_{i}$ and thus in particular $p_{i}$ is an endpoint of $s_{i}$. Now $p_{i} \notin \square$, so $s_{i}$ intersects at least two grid cells.

We continue this walk, for $i=1, \ldots, M$ and consider the following cases (see Figure 6).

(A) There is a segment $s^{\prime} \in \mathcal{Y} \cup\left\{s_{1}, \ldots, s_{i-1}\right\}$, such that $\operatorname{int}\left(s_{i}\right) \cap \operatorname{int}\left(s^{\prime}\right) \neq \emptyset$, see Figure 6(A). This can happen, as the segments of $\mathcal{Y}$ are not necessarily maximal. As $s_{i-1}$ does not hit $\mathrm{s}^{\prime}$ at $\mathrm{p}_{i-1}$, and $\mathrm{s}_{i}$ cannot be extended beyond $\mathrm{p}_{i}$, we must have $\mathrm{s}^{\prime} \subset \mathrm{s}_{i}$. If $\mathrm{s}^{\prime} \in \mathcal{Y}$, then we replace $\mathrm{s}^{\prime}$ by $\mathrm{s}_{i}$ in $\mathcal{Y}$, add the segments of the path constructed so far to $\mathcal{Y}$, and stop the path construction. If $s^{\prime} \in\left\{s_{1}, \ldots, s_{i-1}\right\}$, then we also stop the path construction and add the segments $\left\{\mathrm{s}_{1}, \ldots, \mathrm{s}_{i}\right\} \backslash\left\{\mathrm{s}^{\prime}\right\}$ to $\mathcal{Y}$.

(B) Case (A) does not happen, but $\mathrm{s}_{i}$ hits a segment from $\mathcal{X} \cup \mathcal{Y} \cup\left\{\mathrm{s}_{1}, \ldots, \mathrm{s}_{i-1}\right\}$ at $\mathrm{p}_{i}$, see Figure $6(\mathrm{~B})$. We add the segments $\left\{\mathrm{s}_{1}, \ldots, \mathrm{s}_{i}\right\}$ to $\mathcal{Y}$, and the construction of the path is done.

(C) Cases (A) and (B) do not happen. In this case, $s_{i}$ hits some perpendicular block at $\mathrm{p}_{i}$ (see Figure 6(C)). The algorithm proceeds as before, considering the segment $s_{i}$ and its endpoint $\mathrm{p}_{i}$ instead of $\mathrm{s}_{i-1}$ and $\mathrm{p}_{i-1}$. The conditions of Lemma 4.5 are satisfied, as $\mathrm{s}_{i}$ intersects at least two grid cells. We continue extending the path until one of the cases (A) or (B) happens, or until the number of segments in the path reaches the upper bound of $M$.

Shortcutting the path if it is too long. A more challenging situation occurs when after $M$ steps the path $\mathrm{s}_{1}, \ldots, \mathrm{s}_{M}$ does not hit any segment of $\mathcal{X} \cup \mathcal{Y}$ or itself. To avoid creating an even longer path, the idea is to shortcut the path by adding a single "cheap" segment that connects it to a segment of $\mathcal{X} \cup \mathcal{Y}$. Note that in this case some blocks of $O$ might be cut by the shortcut segment, but we ensure that the total weight of cut blocks from $O$ is negligible. The cut goes along the boundary of some grid cell. An example can be seen in Figure 7.

The proof of Lemma 4.6 below defines a set $\mathcal{E}$ of pairwise disjoint maximal vertical or horizontal segments. The constructed set $\mathcal{E}$ has the following properties:

(A) The segments of $\mathcal{E}$ are contained in the grid edges of $\mathcal{G}$.

(B) Each segment of $\mathcal{E}$ has

(a) one of its endpoints on the segments of $\left\{\mathrm{s}_{1}, \ldots, \mathrm{s}_{M}\right\}$, and

(b) its other endpoint on one of the segments of $X=\mathcal{X} \cup \mathcal{Y} \cup\left\{\mathrm{s}_{1}, \ldots, \mathrm{s}_{M}\right\}$, 


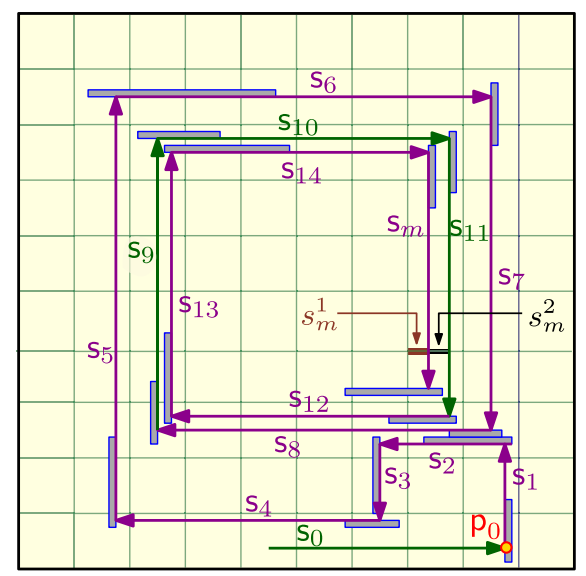

Fig. 7. The construction of the segments $\mathcal{Y}$. The blocks of the considered instance are depicted in gray.

(C) The segments of $\mathcal{E}$ do not intersect any of the segments of $X$ in their interior.

Every segment of $\mathcal{E}$ has an associated weight, which is the total weight of all the blocks that it cuts. The minimum weight segment from $\mathcal{E}$ is the shortcut of the path, and is denoted by $\psi$.

Lemma 4.6. For any path there is always a shortcut of weight of at most $4 w(O) / M$.

Proof. Consider a path $\pi=\mathrm{s}_{0}, \mathrm{~s}_{1}, \ldots, \mathrm{s}_{M}$, and orient the segments of the path such that $\mathrm{s}_{i}$ is oriented toward $\mathrm{s}_{i+1}$, for all $i$. Let $\mathrm{p}_{i}=\mathrm{s}_{i} \cap \mathrm{s}_{i+1}$ for $i=0, \ldots, M-1$, and let $\mathrm{p}_{M}$ be the endpoint of $\mathrm{s}_{M}$ different from $\mathrm{p}_{M-1}$.

By the construction of the path, for any segment $s_{i}$, the two endpoints of $s_{i}$ are in two different cells of $\mathcal{G}$. The $i$ th endpoint of the path, $\mathrm{p}_{i}$, is contained in some grid cell $\square_{i}$, where the path takes either a left turn or a right turn. By Lemma 4.5, we have $\mathrm{p}_{i} \in \operatorname{int}\left(\square_{i}\right)$.

Consider any grid cell $\square$ of $\mathcal{G}$ together with one of its grid edge e, and consider all points $p_{i}$ such that $\mathrm{s}_{i}$ crosses $\mathrm{e}$, and $\mathrm{p}_{i} \in \square$. For each such a point $\mathrm{p}_{i}$ the path first crosses the edge $e$ of $\square$, and then performs a turn to the right or to the left inside $\square$. Let $k=n(\mathrm{e}$, left $)$ and $k^{\prime}=n(\mathrm{e}$, right $)$ denote the total number of such turns to the left and to the right, respectively. Then, $\pi$ crosses $\mathrm{e}$ at least $k+k^{\prime}$ times, and all the intersection points are pairwise distinct. Denote by $\mathrm{p}_{1}^{\prime}, \ldots, \mathrm{p}_{k+k^{\prime}}^{\prime}$ these intersection points, sorted by their position along the edge. Since any two fragments of $\pi$ within $\square$ are disjoint, it must hold that the first $k$ points $\mathrm{p}_{1}^{\prime}, \ldots, \mathrm{p}_{k}^{\prime}$ correspond to the left turns of $\pi$ within $\square$, and the last $k^{\prime}$ points $\mathrm{p}_{k+1}^{\prime}, \ldots, \mathrm{p}_{k+k^{\prime}}^{\prime}$ correspond to the right turns (see the figure).

Let $\bar{p}$ be a point on e that is intersected by a line segment in $\mathcal{X}$. Since e is crossed by at least one vertical segment of $\pi$ such a point must exist. We now break e into a collection $C(\square, \mathrm{e})$ of $k+$ $k^{\prime}$ candidate segments. For each point $\mathrm{p}_{j}^{\prime}$ with $j \in\left\{1, \ldots, k+k^{\prime}\right\}$ we define a point $\mathrm{p}_{j}^{\prime \prime}$ as follows. If $\mathrm{p}_{j}^{\prime}$ lies on the left of $\overline{\mathrm{p}}$, then let $\mathrm{p}_{j}^{\prime \prime}$ denote the leftmost point on $e$ on the right of $\mathrm{p}_{j}^{\prime}$ that is contained in $\mathcal{X} \cup \mathcal{Y}$ or $\pi$. If $\mathrm{p}_{j}^{\prime}$ lies on the right of $\bar{p}$, then let $\mathrm{p}_{j}^{\prime \prime}$ denote the rightmost point on $e$ on the left of $\mathrm{p}_{j}^{\prime}$ that is contained in $\mathcal{X} \cup \mathcal{Y}$ or $\pi$. For each point $\mathrm{p}_{j}^{\prime}$ with $j \in\left\{1, \ldots, k+k^{\prime}\right\}$ we add to $C(\square, \mathrm{e})$ the line segment connecting $\mathrm{p}_{j}^{\prime}$ and $\mathrm{p}_{j}^{\prime \prime}$. Note that all segments in $C(\square, \mathrm{e})$ are pairwise disjoint.

Observe that a grid edge e is contained in up two grid cells

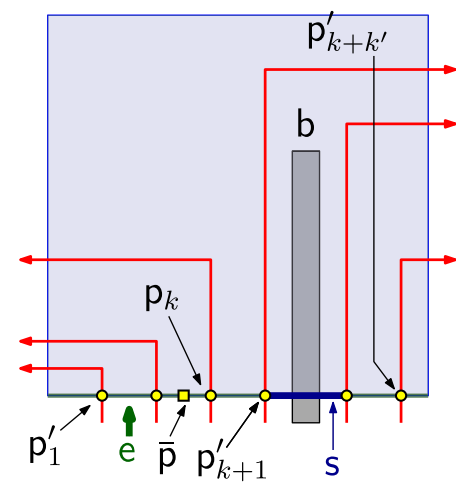
$\square, \square^{\prime}$ (if e lies at the outer boundary then it is contained in only 
one cell). Note that then the sets $C\left(\square\right.$, e) and $C\left(\square^{\prime}\right.$, e) might not be equal. However, note that by construction all candidate segments do not intersect any of the segments of $\mathcal{X} \cup \mathcal{Y}$ and $\pi$ in their interior, either their both endpoints are in $\pi$ or one in $\pi$ and the other one in $\mathcal{X} \cup \mathcal{Y}$. Hence, for two segments $\mathrm{s} \in C(\square, \mathrm{e}), \mathrm{s}^{\prime} \in C\left(\square^{\prime}, \mathrm{e}\right)$ we have that either $\mathrm{s}=\mathrm{s}^{\prime}$ or $\mathrm{s} \cap \mathrm{s}^{\prime}=\emptyset$.

Let $\mathcal{E}$ be the set of all candidate segments for all cells of $\mathcal{G}$ and their corresponding edges. If a grid edge has no turns on it, then naturally it contributes no segments to $\mathcal{E}$.

Consider any candidate segment s obtained while considering a cell $\square$ together with its edge e, and observe that any block of $O$ that is cut by s must end in $\square$, as the turn of the path $\pi$ corresponding to the segment s prevents any block of $O$ crossing s to continue to the next cell. As such, every block of $O$ is cut by at most two segments from the set $\mathcal{E}$.

The size of $\mathcal{E}$ is equal to the number of turns in $\pi$ divided by 2 (i.e., $M / 2$ ), since each segment $s$ might appear in at most two sets $C(\square, \mathrm{e}), \mathrm{s}^{\prime} \in C\left(\square^{\prime}\right.$, e). We defined the weight of a segment $\mathrm{s} \in C$, denoted by $w(\mathrm{~s})$, as the total weight of blocks of $O$ crossed by s. By the above, we have that $\sum_{\mathrm{s} \in C} w(\mathrm{~s}) \leq 2 w(O)$. As such, there is a segment in $\mathcal{E}$ of weight at most $4 w(O) / M$, as claimed.

From the choice of $\psi$ we know that one endpoint of $\psi$ lies on some segment $s_{i} \in\left\{s_{1}, \ldots, s_{M}\right\}$, and another one on some segment $\mathrm{t} \in \mathcal{X} \cup \mathcal{Y} \cup\left\{\mathrm{s}_{1}, \ldots, \mathrm{s}_{i-1}\right\}$.

(A) If $\mathrm{t} \in\left\{\mathrm{s}_{1}, \ldots, \mathrm{s}_{i-1}\right\}$, then adding $\psi$ to the path will create a cycle. We add the segments $\left\{s_{1}, \ldots, s_{i^{\prime}}\right\}$ such that $s_{i^{\prime}}$ is the first segment that is part of the cycle. Then we add the portion of the segments that form the cycle itself (merging added segments that are on the same line and sharing endpoints if necessary). In particular, possibly only a part of $s_{i}$ will be added to the cycle.

(B) If $t \in \mathcal{X} \cup \mathcal{Y}$, then we add the portion of the path until the shortcut, and the shortcut itself, to $\mathcal{Y}$. Again, possibly only a part of $s_{i}$ will be added to the cycle.

This completes the description of the algorithm for computing the decomposition. The resulting set of segments is denoted by $\mathcal{Z}=\mathcal{X} \cup \mathcal{Y}$.

\subsection{Analyzing the Structure of the Resulting Partition}

Here we prove that the construction above partitions the bounding box into faces with a nice structure (i.e., trails and rings, see below), the number of resulting faces is small, and each face has low complexity. This requires quite a bit of care, and the result is summarized in Lemma 4.17p32; the casual reader might want to skip the details on the first reading.

Definition 4.7. A rectilinear polygon $T$ is an $L$-shape if its boundary has exactly six edges.

A rectilinear polygon $T$ with coordinates in $\llbracket N \rrbracket^{2}$ is a narrow polygon if it does not contain any vertex of the grid $\mathcal{G}$ in its interior, and if for any grid cell $\square$ of $\mathcal{G}$ any connected component $\xi$ of $T \cap \square$ (i) is either a rectangle or an $L$-shape and (ii) $\xi$ intersects at most two edges of $\square$.

A narrow polygon is a trail if it has no holes (i.e., it is homotopic to a path). A narrow polygon is a ring if it has a single hole.

4.3.1 Basic Properties of the Construction. The construction immediately implies the following.

Proposition 4.8. The set $\mathcal{X}$ consists of at most $16(1 / \delta)^{2}+4$ segments.

Definition 4.9. A set of segments $\mathcal{Z}^{\prime}$ is nicely connected if (i) no pair of segments of $\mathcal{Z}^{\prime}$ intersect in their interior, and (ii) for any endpoint $p$ of a segment $s \in \mathcal{Z}^{\prime}$ there is a segment $t \in \mathcal{Z}^{\prime}$ perpendicular to $\mathrm{s}$, such that $\mathrm{s} \cap \mathrm{t}=\mathrm{p}$.

Lemma 4.10. The set of segments $\mathcal{Z}=\mathcal{X} \cup \mathcal{Y}$ satisfies the following properties.

(A) $\mathcal{Z}$ is nicely connected. 
(B) We have $|\mathcal{Y}| \leq 1 /(\varepsilon \delta)^{4}$ and $|\mathcal{Z}| \leq 1 /(\varepsilon \delta)^{4}$.

(C) Any segment $\mathrm{s} \in \mathcal{Y}$ that cuts some blocks of $O$ is contained in a single grid edge of $\mathcal{G}$.

(D) The total weight of the blocks of $O$ cut by segments of $\mathcal{Z}$ is bounded by $\varepsilon w(O)$.

(E) Every segment of $\mathcal{X}$ that intersect the interior of the square $[0, N]^{2}$ crosses some grid line of $\mathcal{G}$. Every segment of $\mathcal{Y}$ intersects some grid line of $\mathcal{G}$.

Proof. (A) By construction, no two segments from $\mathcal{Z}$ overlap or intersect in their interior. For each endpoint $\mathrm{p}$ of a segment $\mathrm{s} \in \mathcal{X}$ that does not hit a perpendicular segment from $\mathcal{Z}$ we added a perpendicular segment touching $\mathrm{p}$ to the set $\mathcal{Y}$. The path of segments connecting $\mathrm{p}$ with a segment from $\mathcal{Z}$ is constructed in such a way, that each segment added to $\mathcal{Y}$ has both endpoints touching perpendicular segments from $\mathcal{Z}$. If a segment from $\mathcal{Y}$ gets extended, then it is extended in such a way that the new endpoint touches a perpendicular segment from $\mathcal{Z}$. Thus, the set of segments $\mathcal{Z}$ is nicely connected.

(B) By Proposition $4.8,|X| \leq 16(1 / \delta)^{2}+4$. For each endpoint of a segment of $\mathcal{X}$, except for the four segments forming the boundary of the input square, we added at most $M+1=O\left(1 /\left(\varepsilon \delta^{2}\right)\right)$ segments to the set $\mathcal{Y}$, implying $|\mathcal{Y}|=O\left(1 /\left(\varepsilon \delta^{4}\right)\right)$ and $|\mathcal{Z}|=O\left(1 /\left(\varepsilon \delta^{4}\right)\right)$.

(C) The only segments of $\mathcal{Z}$ that can cut blocks of $O$ are the shortcuts, and each such block has non-empty intersection with some edge of $\mathcal{G}$ (see construction of the shortcuts in the proof of Lemma 4.6).

(D) By Property (C), we only need to bound the total weight of the shortcuts within $\mathcal{Z}$. By Lemma 4.6, each shortcut has a weight of at most $4 w(O) / M$. As each shortcut has been generated while creating a path from an endpoint of a segment from $\mathcal{X}$, and the 4 segments on the boundary do not get extended, there are at most $2(|X|-4)$ shortcuts. The total weight of the blocks of $O$ that have been cut is therefore bounded by $(8(|X|-4) / M) w(O) \leq\left(8\left(16(1 / \delta)^{2}\right) \cdot \varepsilon \delta^{2} / 128\right) w(O) \leq$ $\varepsilon w(O)$, by Proposition 4.8 and Equation $(4.1)_{\mathrm{p} 22}$.

(E) All the segments of $\mathcal{X}$ are long, implying the first part of this claim. By the construction of the segments of $\mathcal{Y}$, each shortcut lies on a single grid edge of $\mathcal{G}$, and all other edges cross or intersect the boundary of the grid cell that contains one of their endpoints in its interior.

On the structure of the connections between segments of $\mathcal{Z}$.

Lemma 4.11. Consider a segment $\mathrm{s} \in \mathcal{Z}$, and let $\square$ be a grid cell such that $\mathrm{s}$ is a reach segment (not necessarily extremal) for an edge e of $\square$, and s does not cross $\square$, see Section 4.2.2 $\mathrm{p21}$. Let $\mathrm{p} \in \square$ be an endpoint of $\mathrm{s}$. Then there exists a segment $\mathrm{t} \in \mathcal{X}$ perpendicular to s such that (i) $\mathrm{s} \cap \mathrm{t}=\mathrm{p}$, (ii) $\mathrm{t}$ does not end at $\mathrm{p}$, and (iii) $\mathrm{t}$ is an extremal reach segment for an edge $\mathrm{e}^{\prime}$ of $\square$ perpendicular to $\mathrm{e}$.

Proof. We assume that $p \in \operatorname{int}(\square)$, since the case where it is on the boundary of the grid cell can be handled in a similar fashion.

By Lemma 4.10, $\mathcal{Z}$ is nicely connected, which implies that there is a segment $t \in \mathcal{Z}$ perpendicular to $s$, such that $s \cap t=p$. Without loss of generality, assume that e is the bottom edge of $\square$. Let $\mathrm{s}_{\max }$ be a maximal segment containing $\mathrm{t}$, which does not intersect any blocks of $O$ or segments of $\mathcal{X}$. We claim that $\mathrm{s}_{\max }$ is the bottom-most reach segment for an edge $\mathrm{e}^{\prime}$ of $\square$, see Figure 8 . As s is a reach segment for e and s does not cross $\square$ (i.e., s does not touch the edge of $\square$ parallel to e), s cannot be extended at $p$. Either $s$ hits $t$ at $p$, or $s$ hits a perpendicular block at $p$. In either case, $s_{\max }$ does not end at $p$.

If $\mathrm{t} \in \mathcal{X}$, then $\mathrm{t}$ is long and so is $\mathrm{s}_{\max }$ (since all the segments of $\mathcal{X}$ are long). If $\mathrm{t} \in \mathcal{Y}$, then $\mathrm{t}$ cannot be a shortcut, since all shortcuts lie on the boundary of grid cells, and $p$ is in the interior of $\square$. Now, by construction, $t$ lies on a long edge of a block, and $s_{\max }$ is at least as long as a block.

The segment $s_{\max }$ is not contained in $\square$, i.e., it intersects an edge $\mathrm{e}^{\prime}$ of $\square$ perpendicular to e (see Figure 8). If $s_{\max }$ crosses $\square$, then it is the bottom-most segment intersecting $\mathrm{e}^{\prime}$ and maximizing the 


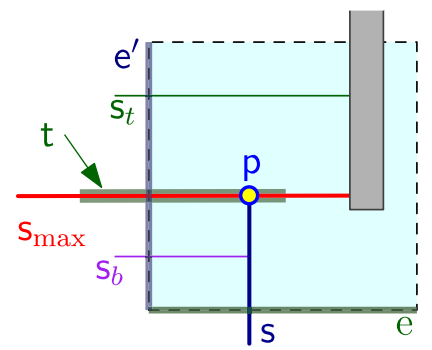

Fig. 8. Illustration of the proof of Lemma 4.11.

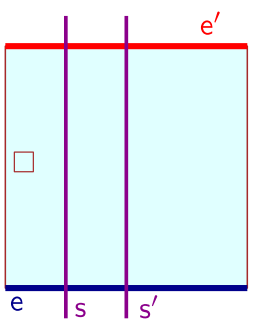

(A)

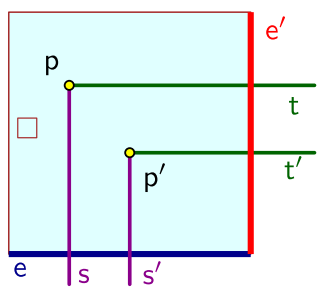

(B)

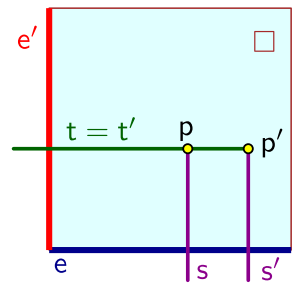

(C)

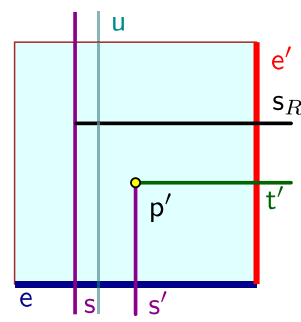

(D)

Fig. 9. Proof of Lemma 4.12.

length of the intersection with $\square$. As such, by construction, it would be in $\mathcal{X}$, thus implying the lemma.

Otherwise, $s_{\max }$ does not cross $\square$. The segment $s_{\max }$ ends in $\square$ by hitting a perpendicular segment from $\mathcal{X}$ or a perpendicular block. This segment or block does not intersect the bottom boundary of $\square$, as it would yield a long segment crossing e, which reaches further than s, which gives a contradiction, as $\mathrm{s}$ is a reach segment for e, see step $(\mathrm{C})_{\mathrm{p} 21}$ in Section 4.2.2. The segment or block hit by $s_{\max }$ crosses the top edge of $\square$ and does not intersect any segments from $\mathcal{Z}$ inside $\square$. Therefore, any segment $\mathrm{s}_{t} \in \mathcal{Z}$ that intersects $\mathrm{e}^{\prime}$ above $\mathrm{s}_{\max } \cap \mathrm{e}^{\prime}$ satisfies $\left\|\mathrm{s}_{t} \cap \square\right\| \leq\left\|\mathrm{s}_{\max } \cap \square\right\|$. Similarly, let $\mathrm{s}_{b} \in \mathcal{Z}$ be a segment which intersects $\mathrm{e}^{\prime}$ below $\mathrm{s}_{\max } \cap \mathrm{e}^{\prime}$. As $\mathrm{s}_{b}$ cannot cross $\mathrm{s}$, and $\mathrm{s}_{\max }$ extends beyond s, we get $\left\|s_{b} \cap \square\right\|<\left\|s_{\max } \cap \square\right\|$.

Thus, $s_{\max }$ is the bottom-most long segment maximizing the length of the intersection with $\square$, and so it is the bottom-most reach segment for $\mathrm{e}^{\prime}$. We get that $\mathrm{s}_{\max } \in \mathcal{X}$, and so $\mathrm{t}=\mathrm{s}_{\max }$.

LEMMA 4.12. Let $\square$ be a grid cell, and let e be an edge of $\square$. Let $\mathrm{s}, \mathrm{s}^{\prime} \in \mathcal{Z}$ be two segments that cross the edge e, such that there is no segment $\mathrm{s}^{\prime \prime} \in \mathcal{Z}$ which intersects e between these two crossings. Then, there is an edge $\mathrm{e}^{\prime} \neq \mathrm{e}$ of $\square$ and segments $\mathrm{t}, \mathrm{t}^{\prime} \in \mathcal{Z}$ intersecting $\mathrm{e}^{\prime}$, such that $\mathrm{s} \cap \mathrm{t} \neq \emptyset$ and $\mathrm{s}^{\prime} \cap \mathrm{t}^{\prime} \neq \emptyset$ ( where possibly $\mathrm{s}=\mathrm{t}$ or $\mathrm{s}^{\prime}=\mathrm{t}^{\prime}$ ).

Proof. We assume w.l.o.g. that e is the bottom edge of $\square$, $\mathrm{s}$ is to the left of $\mathrm{s}^{\prime}$, and that $\|\mathrm{s} \cap \square\| \geq$ $\left\|s^{\prime} \cap \square\right\|$. There are now several possible cases.

(A) $s$ and $s^{\prime}$ intersect the top edge of $\square$ : The claim holds for $t=s$ and $t^{\prime}=s^{\prime}$, see Figure $9(\mathrm{~A})$.

(B) $\mathbf{s}$ and $\mathbf{s}^{\prime}$ both have endpoints inside $\square$ : Let $\mathrm{p}$ and $\mathrm{p}^{\prime}$ be the two endpoints of $\mathrm{s}$ and $s^{\prime}$ in $\square$, respectively. By Lemma 4.10 (A), there are two segments $t$ and $t^{\prime}$ in $\mathcal{Z}$ that are perpendicular to $s$ and $s^{\prime}$, respectively, such that $s \cap t=p$ and $s^{\prime} \cap t^{\prime}=p^{\prime}$. The segments $t$ and $t^{\prime}$ are not contained in int $(\square)$, by Lemma 4.10 (E). As such, each of them intersects an edge of $\square$. 


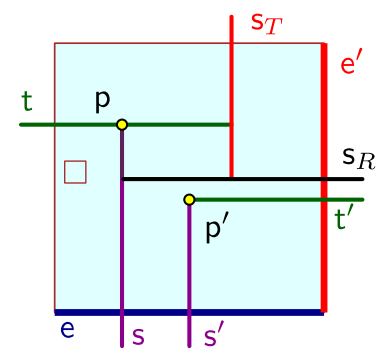

Fig. 10. Illustration for Lemma 4.12. case (B.c).

(a) If $t$ intersects the right edge of $\square$, then $t^{\prime}$ also intersects the same edge, as otherwise, either $t^{\prime}$ would cross s, or t would cross s'. See Figure 9(B).

(b) If $\mathrm{t}^{\prime}$ intersects the left edge of $\square$, then, by the assumption that $\|\mathrm{s} \cap \square\| \geq\left\|\mathrm{s}^{\prime} \cap \square\right\|$, we have that $\|s \cap \square\|=\left\|s^{\prime} \cap \square\right\|$ and $t=t^{\prime}$, implying the claim. See Figure $9(\mathrm{C})$.

(c) Otherwise, $t$ intersects only the left edge of $\square$, and t' intersects only the right edge of $\square$, see Figure 10. As there are no edges in $\mathcal{Z}$ intersecting e between $s$ and $\mathrm{s}^{\prime}$, and by assumption $\|\mathrm{s} \cap \square\| \geq\left\|\mathrm{s}^{\prime} \cap \square\right\|$, it follows that $\mathrm{s}$ is a reach segment of e. By Lemma 4.11, $\mathrm{t}$ is a reach segment for the left edge of $\square$ (specifically, the bottom-most reach segment), and it does not end at $p$.

The segment $t$ does not touch the right edge of $\square$, as otherwise the claim holds. Now, by applying Lemma 4.11 to $t$, we have that $t$ hits a perpendicular reach segment $\mathrm{s}_{T}$ in $\square$. The segment $\mathrm{s}_{T}$ does not intersect the bottom edge e of $\square$, as otherwise $\| s_{T} \cap$ $\square\|>\| \mathrm{s} \cap \square \|$, and that would contradict s being a reach segment for e. Thus, $\mathrm{s}_{T}$ must intersect the top edge of $\square$ and has an endpoint inside $\square$. Applying Lemma 4.11 to $s_{T}$ in turn, implies that it hits a perpendicular reach segment $\mathrm{s}_{R}$ that must intersect the right edge of $\square$. If $\mathrm{s}_{R} \neq \mathrm{t}^{\prime}$ then, by Lemma $4.11, \mathrm{~s}_{R}$ hits another reach segment that crosses e, but this reach segment must be $\mathrm{s}$, see Figure 10. The case that $\mathrm{s}_{R}=\mathrm{t}^{\prime}$ follows verbatim by the same analysis, by observing that $\mathrm{t}^{\prime}$ does not hit $\mathrm{s}^{\prime}$ (i.e., $\mathrm{t}^{\prime}$ hitting a segment $\mathrm{t}^{\prime \prime}$ implies that an endpoint of $\mathrm{t}^{\prime}$ is in the interior of $\left.\mathrm{t}^{\prime \prime}\right)$. For the pairs $\mathrm{s}, \mathrm{s}_{R}$ and $\mathrm{s}^{\prime}, \mathrm{t}^{\prime}$ the claim now follows.

(C) $\mathbf{s}$ intersects the top edge of $\square$, and $\mathbf{s}^{\prime}$ does not: By Lemma 4.10, there is a segment $t^{\prime} \in$ $\mathcal{Z}$ that is perpendicular to $\mathrm{s}^{\prime}$ at its endpoint $\mathrm{p}^{\prime} \in \square$, and furthermore $\mathrm{t}^{\prime}$ is not contained in $\operatorname{int}(\square)$. As $s$ is to the left of $s^{\prime}$, the segment $t^{\prime}$ intersects the right edge $e^{\prime}$ of $\square$ (see Figure $9(\mathrm{D})$ ). Let $\mathrm{s}_{R}$ be a reach segment for $\mathrm{e}^{\prime}$. Such a segment exists, as the maximal segment containing $\mathrm{t}^{\prime}$ is a candidate to be a reach segment for $\mathrm{e}^{\prime}$.

We claim that $\mathrm{s}_{R}$ touches $\mathrm{s}$. If $\mathrm{s}_{R}$ crosses $\square$, then $\mathrm{s}_{R}$ must touch $\mathrm{s}$ (and either $\mathrm{s}$ or $\mathrm{s}_{R}$ goes along an edge of $\square$ ). If $\mathrm{s}_{R}$ does not cross $\square$, then, by Lemma 4.11, $\mathrm{s}_{R}$ hits a perpendicular reach segment $u$ in $\square$. The segment $u$ is a vertical reach segment, and must be as long as s inside $\square$; that is, it must cross $\square$. However, there is no segment in between s and s' crossing e, which implies that $\mathrm{s}=\mathrm{u}$. We conclude that $\mathrm{s}_{R}$ intersects $\mathrm{s}$, as desired.

4.3.2 Faces of the Partition. The segments of $\mathcal{Z}$ subdivide the input square into a collection of faces that are the connected components of $[0, N]^{2} \backslash \cup \mathcal{Z}$ (as such, the faces are open sets), where $\cup \mathcal{Z}=\cup_{s \in Z^{s}}$. Let $\mathcal{F}$ denote the set of faces of this partition, and $\mathcal{F}_{+}$the set of faces that contain at least one block of $O$. Our purpose here is to prove that the number of resulting faces is bounded by a constant and that each face is either a trail or a ring.

Inside a grid cell, faces are rectangles or L-shaped. 


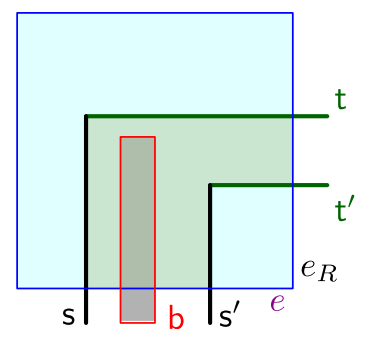

Fig. 11. A connected component of a face $\Xi \in \mathcal{F}_{+}(\mathcal{Z})$ within a grid cell (denoted by a shaded area) must have a simple shape, i.e., it is either a rectangle or an $L$-shape.

ObSERVATion 4.13. No face of $\mathcal{F}_{+}$contains a vertex of $\mathcal{G}$ in its interior.

Lemma 4.14. Consider a face $\Xi \in \mathcal{F}_{+}$and let $\square$ be a grid cell with $\Xi \cap \square \neq \emptyset$. Consider a connected component $\xi$ of $\Xi \cap \square$. Then $\operatorname{int}(\xi)$ is the interior of a rectangle or the interior of an L-shape. Also, $\xi$ has non-empty intersection with at most two edges of $\square$.

Proof. Consider the case when $\xi$ has non-empty intersection with some block b $\in O$ contained in $\Xi$. Let e be an edge of $\square$ such that e $\cap \operatorname{int}(b) \neq \emptyset$, and assume w.l.o.g. that e is the bottom edge of $\square$ (see Figure 11). Let $s, s^{\prime} \in \mathcal{Z}$ be $L$-segments that intersect $\square$ and intersect e at some points $p_{s}$ and $p_{s^{\prime}}$, respectively, such that $p_{s}$ is to the left of $e \cap \operatorname{int}(b), p_{s^{\prime}}$ is to the right of e $\cap \operatorname{int}(b)$, and no segment from $\mathcal{Z}$ which intersects $\square$ touches e in between $p_{s}$ and $p_{s^{\prime}}$. Such segments exist, as no segment from $\mathcal{Z}$ intersects e inside e $\cap$ int(b), the leftmost long segment intersecting $\square$ and touching e (which belongs to $\mathcal{X}$ ) either contains the left edge of $\mathrm{b}$ or is to the left of it, and the rightmost long segment intersecting $\square$ and touching e (which also belongs to $\mathcal{X}$ ) either contains the right edge of $b$ or is to the right of it.

Parts of the segments s, $s^{\prime}$ lie on the boundary of $\xi$. Denote by $t$ and $t^{\prime}$ the lines given by applying Lemma 4.12 to $s$ and $s^{\prime}$. If $t$ and $t^{\prime}$ both intersect the top edge of $\square$, then the claim follows and $\xi$ is a rectangle. Otherwise, assume w.l.o.g. that both $\mathrm{s}$ and $\mathrm{s}^{\prime}$ intersect the right edge $\mathrm{e}_{R}$ of $\square$, and assume that $\mathrm{t}$ is the bottom-most segment touching $\mathrm{s}$ and $\mathrm{e}_{R}$ and $\mathrm{t}^{\prime}$ is the topmost segment touching $\mathrm{s}^{\prime}$ and $\mathrm{e}_{R}$. From Lemma 4.10 the set of lines $\mathcal{Z}$ is nicely connected, and by construction, all lines in $\mathcal{Z}$ with non-empty intersection with int $(\square)$ for some grid cell $\square$ touch the boundary of $\square$. Hence, there can be no segment of $\mathcal{Z}$ within $\square$ intersecting $\mathrm{e}_{R}$ between $\mathrm{e}_{R} \cap \mathrm{t}$ and $\mathrm{e}_{R} \cap \mathrm{t}^{\prime}$, and the claim follows.

The above proves the claim for any $\xi$ which has non-empty intersection with some block $\mathrm{b} \in O$ contained in $\Xi$. However, there can potentially be a grid cell $\square$ and a face $\Xi$ such that a connected component $\xi$ of $\Xi \cap \square$ intersects no blocks of $O$. The proof in this case follows by propagating the property to the adjacent grid cells of $\square$ intersecting $\Xi$ : Consider a connected component $\xi^{\prime}$ of $\Xi \cap \square^{\prime}$, such that $\xi \cap \xi^{\prime} \neq \emptyset$ and assume that the claim holds for $\xi$ (since $\Xi \in \mathcal{F}_{+}$there must be one cell $\square$ such that $\square \cap \Xi \neq \emptyset$ ).

If $\xi^{\prime}$ intersects a block of $O$, then the claim follows by the above. So assume that $\xi^{\prime}$ does not intersect any block of $O$. Consider the segments of $\mathcal{X}$ clipped to $\operatorname{int}\left(\square^{\prime}\right)$.

Each segment $\mathcal{X}$ clipped to int $\left(\square^{\prime}\right)$ either crosses $\square^{\prime}$, or it has a loose end inside $\square^{\prime}$. In the second stage of the construction, every such loose end is connected up to a path of segments, importantly by a segment that leaves the

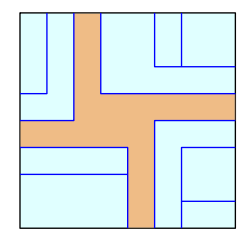
interior of the cell. Then, during the second stage, a path might end up hitting an existing segment. Thus, the set of segments of $\mathcal{Z}$ clipped to the interior of $\square^{\prime}$ is formed by the union of segments that cross the cell and $L$-shaped curves with their endpoints on the boundary of $\square^{\prime}$. None of these 
curves cross each other inside $\square^{\prime}$, although they might have a non-empty intersection. See the figure on the right for an example.

Consider a partition of $\square^{\prime}$ that might be formed by such a collection of $L$-shaped curves. Consider a face $\xi^{\prime}$ in such a partition of $\square^{\prime}$ which is not a rectangle. Then it must have a corner $p$, such that the angle inside $\xi^{\prime}$ is $270^{\circ}$. The only way such a corner can be formed is because one of the segments adjacent to $\mathrm{p}$ hits a block of $O$. But that implies that $\operatorname{int}\left(\xi^{\prime}\right)$ intersects a block of $O$, and as such, by the above it is $L$-shaped and in particular it has non-empty intersection with at most two edges of $\square$. By Observation 4.13, the latter holds also when $\xi^{\prime}$ is a rectangle.

Shortcuts are anchored at segments visiting both cells.

Lemma 4.15. Let $\square$ and $\square$ ' be two neighboring grid cells and let $\mathrm{e}=\square \cap \square$ '. Let $\mathrm{t} \subseteq$ e be a maximal segment of $\mathcal{Z}$ contained in $\mathrm{e}$, and assume that $\mathrm{t}$ does not contain an endpoint of $\mathrm{e}$. Then $\mathrm{t}$ is incident with segments $\mathrm{s}, \mathrm{s}^{\prime} \in \mathcal{Z}$ (where possibly $\mathrm{s}=\mathrm{s}^{\prime}$ ) such that $\mathrm{s}$ intersects $\operatorname{int}(\square)$ and $\mathrm{s}^{\prime}$ intersects $\operatorname{int}\left(\square^{\prime}\right)$.

Proof. From the construction of $\mathcal{X}$ and $\mathcal{Y}$, the segment s consists of one or multiple shortcut segments, as any other segment from $\mathcal{Z}$ would touch an endpoint of e.

Let $\mathrm{u} \subseteq \mathrm{t}$ be the first shortcut added to $\mathcal{Y}$. There are two segments $\mathrm{s}, \mathrm{s}^{\prime} \in \mathcal{Y} \cup \mathcal{X}$ such that the endpoints of $\mathrm{u}$ are contained in these two segments. Assume s intersects int $(\square)$.

If $\mathrm{s}$ also intersects int $\left(\square^{\prime}\right)$, then the claim holds. Similarly, if $s^{\prime}$ intersects int $\left(\square^{\prime}\right)$, then we are done. So, it must be that $s^{\prime}$ intersects int $(\square)$, and both $s$ and $s^{\prime}$ have an endpoint on e; see figure on the right. Let $p$ (respec-

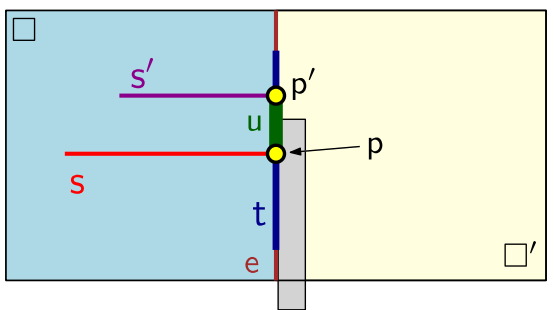
tively, $p^{\prime}$ ) be the endpoint of s (respectively, $s^{\prime}$ ) on e. We have that s does not hit a perpendicular block at $\mathrm{p}$, as otherwise this would induce a leftmost long segment crossing the top or the bottom edge of $\square^{\prime}$. In turn, such a segment, by construction, is in $\mathcal{X}$, see $(\mathrm{A})_{\mathrm{p} 21}$ in Section 4.2.2. This would contradict the assumption that $\mathrm{t}$ does not contain an endpoint of e. Similarly, s cannot hit any vertical segment of $\mathcal{X}$ at $p$, since all such segments are long,

So, it must be that $\mathrm{s}$ is in $\mathcal{Y}$, and furthermore, it got shortened when the shortcut u was created (because this is the only way for s to have an endpoint on e). We can apply verbatim the same logic to $s^{\prime}$. However, by construction, it is not possible that when the shortcut u was introduced between $s$ and $s^{\prime}$, both of them got clipped. ${ }^{9}$

Faces of $\mathcal{F}_{+}$do not fork. Now we study the structure of the faces in $\mathcal{F}_{+}$at the boundary of the grid cells. In the following lemma, we show that multiple connected components of a face inside one grid cell $\square^{\prime}$ cannot merge into one component in a neighboring grid cell $\square$.

Lemma 4.16. Let $\square$ and $\square^{\prime}$ be two grid cells sharing a common edge e. Consider a face $\Xi \in \mathcal{F}_{+}$ such that $\Xi \cap \square \neq \emptyset$, and let $\xi$ be a connected component of $\Xi \cap \square$ such that $\xi \cap \mathrm{e} \neq \emptyset$. Then there is exactly one connected component $\xi^{\prime}$ of $\Xi \cap \square^{\prime}$ such that $\xi \cap \xi^{\prime} \neq \emptyset$.

Proof. We remind the reader that the grid cells are closed sets, but faces of $\mathcal{F}_{+}$are open sets. Let $\mathrm{p} \in \xi \cap$ e. Let $\xi^{\prime}$ be a connected component of $\Xi \cap \square^{\prime}$ containing p. Clearly, $\mathrm{p} \in \xi \cap \xi^{\prime} \neq \emptyset$.

We claim that $\xi^{\prime}$ is unique. Assume otherwise, i.e., that there are two connected components $\xi_{1}^{\prime}$ and $\xi_{2}^{\prime}$ of $\Xi \cap \square^{\prime}$ which have non-empty intersection with $\xi$ (and they are consecutive along e). Let $\mathrm{t} \in \mathcal{Z}$ be a segment intersecting $\operatorname{int}\left(\square^{\prime}\right)$, such that $\mathrm{t}$ intersection with e is between $\xi_{1}^{\prime} \cap \xi$ and $\xi_{2}^{\prime} \cap \xi$ (see Figure 12). Such a segment exists, as $\xi_{1}^{\prime} \cap \square^{\prime}$ and $\xi_{2}^{\prime} \cap \square^{\prime}$ are not connected.

\footnotetext{
${ }^{9}$ Underlying our argument here is the monotonicity of $\mathcal{Y}$ : As the construction continues, the union of segments in this set only grows.
} 


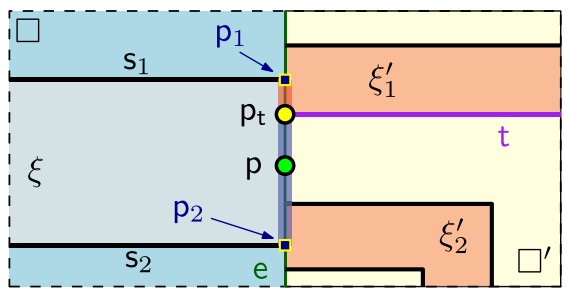

Fig. 12. Illustration of the proof of Lemma 4.16.

Let $s_{1}, s_{2} \in \mathcal{Z}$ be the segments intersecting $\square$, touching e and bounding $\xi \cap \square$. Then there is no segment $\mathrm{s}_{3} \in \mathcal{Z}$ which intersects the interior of $\square$ and intersects e between $\mathrm{p}_{1}=\mathrm{s}_{1} \cap$ e and $\mathrm{p}_{2}=$ $\mathrm{s}_{2} \cap \mathrm{e}$. As $\mathrm{p}_{\mathrm{t}}=\mathrm{t} \cap \mathrm{e}$ is between $\xi_{1}^{\prime} \cap \xi$ and $\xi_{2}^{\prime} \cap \xi$, it holds that $\mathrm{p}_{\mathrm{t}}$ is between $\mathrm{p}_{1}$ and $\mathrm{p}_{2}$. Implying that $t$ ends at $p_{t} \in e$, and does not intersect the interior of $\square$.

We claim that either $p_{t} p_{1}$ or $p_{t} p_{2}$ is contained in $\mathcal{Z}$, which contradicts that either $\xi \cap \xi_{1} \neq \emptyset$ or $\xi \cap \xi_{2} \neq \emptyset$. Hence, the component $\xi^{\prime}$ is unique.

As $p_{t}$ is an endpoint of $t, t$ touches a perpendicular segment from $\mathcal{Z}$ at $p_{t}$. Let $s$ be a maximal segment, such that (i) $s$ is contained in e, (ii) $s$ contains $p_{t}$, and (iii) $s$ is contained in $\mathcal{Z}$. From Lemma 4.15 segment $\mathrm{s}$ contains an endpoint of e, or $\mathrm{s}$ is incident with a segment intersecting $\operatorname{int}(\square)$. In either case, one of the segments $p_{t} p_{1}, p_{t} p_{2}$ is contained in s, i.e., it is contained in $\mathcal{Z}$.

Faces of $\mathcal{F}_{+}$are either trails or rings.

Lemma 4.17. A face $\Xi$ of $\mathcal{F}_{+}$is either a trail or a ring, see Definition $4.7_{\mathrm{p} 25}$. The number of faces of $\mathcal{F}_{+}$is $O\left(1 /\left(\delta \varepsilon^{2}\right)\right)$, and each face has $1 /(\varepsilon \delta)^{4}$ vertices.

Proof. Consider a face $\Xi$. Consider the set of all connected components of $\Xi$ when clipped to the grid cells of $\mathcal{G}$; that is, $\mathcal{C}=\{\Xi \cap \square \mid \square$ cell of $\mathcal{G}$, and $\Xi \cap \square \neq \emptyset\}$. Let $\mathrm{G}=(\mathcal{C}, \mathrm{E})$, where $\xi \rho \in \mathrm{E}$, if $\xi, \rho \in \mathcal{C}$ and $\xi \cap \rho \neq \emptyset$. By definition the graph $\mathrm{G}$ is connected. By Lemma 4.14 and Lemma 4.16, all the vertices of $G$ are either of degree one or two. That implies that $G$ is either a cycle or a path.

As for the number of faces, consider the construction of $\mathcal{Z}$, just before $\mathcal{Y}$ is computed. At this stage, there are $O(|X|)$ faces. Now, every endpoint of a segment of $\mathcal{X}$, might give rise to one new face during the construction of $\mathcal{Y}$. As such, the total number of faces is bounded by $O(|X|)$. The later part of the claim now follows by Lemma $4.10_{\mathrm{p} 26}$.

Note that we do not need to care about faces in $\mathcal{F} \backslash \mathcal{F}_{+}$, since they do not contain any blocks from the optimal solution.

\subsection{The Approximation Algorithm}

The basic idea of the algorithm is to start from the decomposition of Section 4.2 of the input into trails and rings, see Lemma 4.17. We show that for the case of trails, one can compute the independent set inside them optimally. We also show that rings can be decomposed into collections of trails, with only a small loss in the objective. Combining the two algorithms results in the desired approximation algorithm. Finally, we show how to adapt the resulting algorithm to handle large rectangles (and not only blocks).

\subsubsection{Computing the Maximum Weight Independent Set of Blocks Inside a trail.}

Trails can be recursively divided without cutting any blocks.

LEMmA 4.18. Consider a trail T whose boundary has $k$ vertices, for $k \geq 4$. Let $O$ be an independent set of blocks contained in $T$, such that $|O| \geq 2$. Then, there are two trails $T_{1}$ and $T_{2}$, with non-empty 


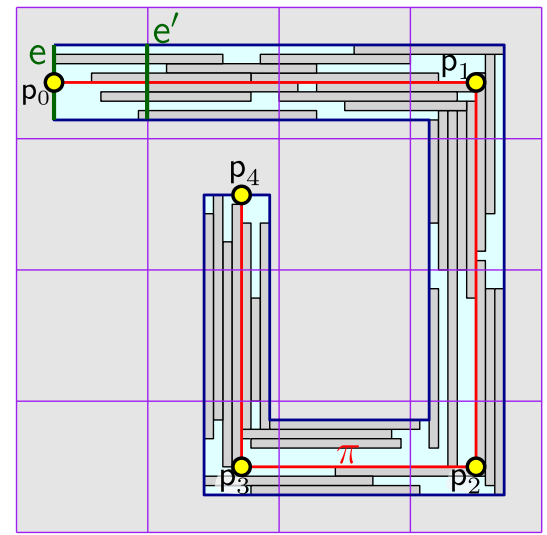

Fig. 13. Illustration of the proof of Lemma 4.18.

interior, each with at most $k$ vertices, such that $T=T_{1} \cup T_{2}$, and every block of $O$ is contained either in $T_{1}$ or $T_{2}$.

Proof. Consider a segment s with integer coordinates, such that (i) $\operatorname{int}(\mathrm{s}) \subseteq \operatorname{int}(T)$, (ii) both endpoints of $\mathrm{s}$ are on the boundary of $T$, and (iii) $\mathrm{s}$ does not intersect the interior of any block of $O$. A segment s with the above properties is a cut segment for $T$. Clearly, if such a segment exists, we cut $T$ along s, and the claim holds.

We now assume that there is no cut segment, i.e., $T$ can not be shrank any further. Arguing as in Lemma 4.17, there must be a grid cell $\square$, and a connected component $\xi$ of $\square \cap T$, which is connected to the rest of the trail through a single segment, denoted by $\mathrm{e}^{\prime}$, on the boundary of $\square$, and assume without limiting generality that $\mathrm{e}^{\prime}$ is vertical, and on the right side of $\square$. By Lemma 4.14, the polygon $\xi$ is either a rectangle or an $L$-shaped polygon. If $\xi$ is $L$-shaped, then consider the maximal rectangle $\mathrm{r} \subseteq \xi$ that has $\mathrm{e}^{\prime}$ as its right side. Any block $\mathrm{b} \in O$ that intersects the interior of $\xi$, must intersect $\mathrm{e}^{\prime}$, as it is the only way from $\xi$ in this grid cell. As such, $\mathrm{b} \cap \xi \subseteq \mathrm{r}$. We conclude, that as far as the optimal solution, we can replace $\xi$ by $r$ in $T$. But this imply that there is a cut segment (i.e., the edge separating $r$ from the rest of $\xi$ ). See Figure 13.

Thus, the polygon $\xi$ is a rectangle, its interface to the rest of $T$ is through the edge $\mathrm{e}^{\prime}$, and let $\mathrm{e}$ be edge of $\xi$ parallel to $\mathrm{e}^{\prime}$.

Assume for the time being that e has length larger than 1 , and let $\mathrm{p}_{0}$ be a point with integer coordinates in the interior of e. Shoot a ray from $p$ into the interior of $T$. This ray must hit a perpendicular block of $\boldsymbol{O}$ at a point $\mathrm{p}_{1}$, and as in construction of $\boldsymbol{Y}$ (see Section 4.2.3 $\mathrm{p}_{22}$ ). We continue the ray shooting from $p_{1}$ along the edge of $b$. Since $T$ is narrow, and inside a grid cell of $\mathcal{G}$ each connected component of $T$ is either a rectangle or is $L$-shaped (see Lemma 4.14), it follows that the generated path must end at an edge of $T$. The generated path $\pi$ does not intersect the interior of any block of $O$, its two endpoints are on the boundary of $T$, and it cuts $T$ into two rectilinear polygons with at most $k$ vertices each.

Add figure for the next paragraph.

The case that $\mathrm{e}$ is of length one requires a special handling. If the trail is a single rectangle, then the claim trivially holds, as it contains the blocks inside it in a linear order, and one can easily cut the trail after the first block. Otherwise, the trail must have a turn in it as it is being traversed from $\xi$. Assume, without limiting generality, that this is a right turn, and perform a ray shooting, as above from the bottom endpoint of e. If the ray shooting cut across $T$, without cutting any block, then we have a cut segment. Otherwise, it must have hit a vertical block $b$ at a point $p_{0}$. If $p_{0}$ is a 
vertex of $T$, then it is easy to verify that one can cut $T$ by a vertical segment through $\mathrm{p}_{0}$. As such, it must be that $\mathrm{p}_{0}$ is in the interior of $T$, and the argument above for the interior ray shooting applies, as can be easily verified, and it implies the claim.

The approximation algorithm for trails.

LEMmA 4.19. Let $N, \varepsilon$ and $\delta$ be parameters as above, let $\mathcal{B}$ be a set of $m$ weighted ( $\delta$-large) blocks with vertices in $\llbracket N \rrbracket^{2}$, and let $k$ be a parameter. Let $Q$ be the set of all possible trails within $\llbracket N \rrbracket^{2}$ with at most $k$ vertices each. For a trail $T \in Q$, let $W_{\text {opt }}(T)$ be the weight of the maximum weight independent set of blocks of $\mathcal{B}$. Then, one can compute exactly the value of $W_{\text {opt }}(\cdot)$ for all the trails of $Q$ in time $O\left(N^{4 k} m k \log k\right)$.

Proof. Let $\mathcal{P}$ be the set of all rectilinear polygons with at most $k$ vertices from the set $\llbracket N \rrbracket^{2}$. An easy calculation shows that $|\mathcal{P}| \leq 2 N^{2 k}$. We verify for every polygon in $\mathcal{P}$ that it is a trail, and if not, we reject it. Let $Q$ be the resulting set of trails, Clearly, verifying if a polygon of $\mathcal{P}$ is a trail can be done in $O(k N \log (k N))$ time. As such, computing $Q$ takes $O\left(k N^{2 k+1} \log (k N)\right)$ time.

Now, for every trail $T \in Q$, we compute the value of $W_{\text {opt }}(T)$. First, we check for the maximum weight single block contained in $T$. Next, we consider the possibility that the optimal solution within $T$ consists of more than one block. From Lemma 4.18, $T$ can then be broken into two smaller trails $T_{1}, T_{2}$, each with at most $k$ vertices, such that the optimal solution for $T$ is a union of the optimal solutions for $T_{1}$ and $T_{2}$. A such, we try all such partitions (naively, there are $|Q|$ such partitions), and for each such partition we verify that it is valid, and then we compute the best solution from all such possibilities.

Given $T$ and $T_{1}$, computing $T_{2}$ can be done in $O(k \log k)$ time using sweeping. As such, the resulting algorithm has running time $O\left(|Q|^{2} k \log k\right)$.

4.4.2 Computing the Maximum Weight Independent Set of Blocks Inside a Ring. The situation here is somewhat more involved. As before, if one can break the ring into two smaller rings, or into a ring and trail, without intersecting any blocks from the optimal solution, the algorithm will try this partition. Alternatively, it will perform a decomposition into a ring and a trail, where there would be a certain (small) loss in the objective.

Rings can be divided without too much loss.

Lemma 4.20. Consider a ring $\sigma$ with $k$ vertices, for $k \geq 4$. Let $O$ be an independent set of blocks contained in $\sigma$, such that $|O| \geq 2$. Then, one the following holds.

(A) There is a trail $T$ such that $\operatorname{cl}(T)=\operatorname{cl}(\sigma)$ with $O(k / \varepsilon)$ vertices, such that $O \subseteq T$.

(B) There are two interior disjoint polygons $\sigma_{1}$ and $\sigma_{2}$, with at most $k$ vertices each, such that $\sigma=\sigma_{1} \cup \sigma_{2}$ and such that $\sigma_{1}$ and $\sigma_{2}$ do not cut any block of $O$. Furthermore, each of the two polygons is either a trail or a ring.

(C) There are interior disjoint trails $T_{1}, \ldots, T_{m}$, such that:

(I) $\bigcup_{i} T_{i}=\sigma$.

(II) The blocks of $O$ cut by the boundaries of $T_{1}, \ldots, T_{m}$ have a total weight of at most $(\varepsilon / 4) w(O)$.

(III) Every trail $T_{i}$ has $O(1 / \varepsilon)$ vertices.

(IV) For any $i, \cup_{j=i+1}^{m} T_{j}$ is a ring (or a trail) with $k+O(1)$ vertices.

Proof. Consider any edge e of the grid $\mathcal{G}$, that intersects $\sigma$, and the segments of $\sigma \cap$ e. If any of these segments does not intersect a block of $O$, then we cut $\sigma$ along it, ending up with a trail. We obtain case (A).

Otherwise, let $x_{0}$ be the minimum $x$-coordinate of any point in $\sigma$, and let $\mathrm{p}^{\prime}=\left(x_{0}, y_{0}\right)$ be the bottom-most vertex of $\sigma$ with $x$-coordinate equal to $x_{0}$. Let $\square$ be the grid cell of $\mathcal{G}$ containing $\mathrm{p}^{\prime}$, 


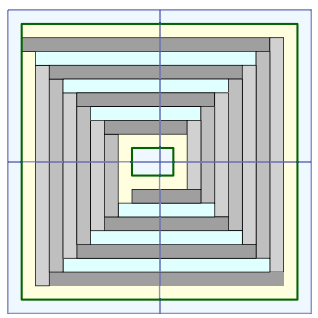

(A)

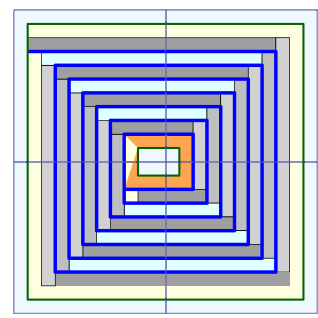

(B)

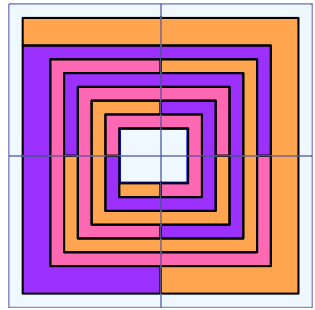

(C)

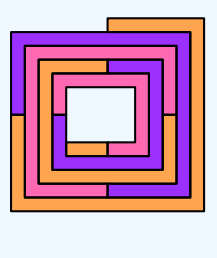

(D)

Fig. 14. (A) A ring with the set of blocks $O$. (B) The resulting decomposition into a trail and a smaller ring. (C) Breaking the long trail into shorter trails. (D) The union of a suffix of these trails forms a ring (or a trail).

and let $\xi$ be the connected component of $\sigma \cap \square$ that has $p^{\prime}$ as a vertex. The polygon $\xi$ is $L$-shaped, and let $\mathrm{e}_{t}$ and $\mathrm{e}_{r}$ be its top and right edges, respectively.

Let $\mathcal{B}_{\xi}$ be the set of all blocks of $O$ crossing either $\mathrm{e}_{t}$ or $\mathrm{e}_{r}$. Let $\mathrm{b} \in \mathcal{B}_{\xi}$ be the block with minimum $L_{1}$-distance from $\mathrm{p}^{\prime}$, and assume w.l.o.g. that $\mathrm{b}$ intersects $\mathrm{e}_{r}$. Let $\mathrm{s}$ be the top edge of $\mathrm{b}$. Extend $\mathrm{s}$ to the left until it hits the outer boundary of $\xi$, and denote this endpoint of $\mathrm{s}$ by $\mathrm{p}_{0}$. Note that after extending s does not cut any blocks of $O$, as $b$ is the closet block to $\mathrm{p}^{\prime}$.

The point $\mathrm{p}_{0}$ lies in the interior of an edge of $\square$, and in the interior of an edge of $\sigma$. As in Lemma 4.18, compute a path $\pi$ in $\sigma$ by shooting a ray from $\mathrm{p}_{0}$ along $\mathrm{s}$, and turning whenever the ray hits a block of $O$. This process terminates when one of the following happens.

(I) The path $\pi$ hits the outer boundary of $\sigma$. In this case, it is easy to verify that this splits $\sigma$ into a trail and a ring, without breaking any block in the process, and both the trail and the ring have at most $k$ vertices each. This corresponds to case (B).

(II) The path $\pi$ hits itself. Let $C$ be the portion of the loop formed by this path, and observe that $C$ has at most $k / 2$ vertices, as can be easily verified, and $C$ breaks $\sigma$ into two rings, as desired. This is again case (B).

The only remaining possibility is that the path $\pi$ hits the inner boundary of $\sigma$. Cutting $\sigma$ along $\pi$, we obtain a new trail polygon $\phi$, which potentially can have a large number of vertices (i.e., $\phi$ is a spiral folded over itself). See Figure 14.

If $\phi$ has $O(k / \varepsilon)$ vertices, then we are done, as this is case (A). Otherwise, let $\xi_{1}, \ldots, \xi_{m}$ be the connected components of $\{\square \cap \phi \mid \square \in \mathcal{G}\}$ that are $L$-shaped in their order along $\phi$. Each such connected component has at most two edges intersecting the interior of $\phi$, and let $S=\left\{\mathrm{s}_{1}, \ldots, \mathrm{s}_{2 m}\right\}$ be the segments that corresponds to these edges, again, in their order along $\phi$.

Consider the "ladder" $L_{i}=\left\{\mathrm{s}_{i+t}, \mathrm{~s}_{i+2 t}, \mathrm{~s}_{i+3 t}, \ldots\right\}$ for $t=\lceil 8 / \varepsilon\rceil$. Every block of $O$ intersects at most two of segments of $\mathrm{S}$. As such, there is a choice of $i$, such that the weight of $L_{i}$ is at most $(\varepsilon / 4) w(O)$. We cut $\phi$ along these edges, creating the desired decomposition of $\sigma$ into trails. This is case $(C)$, and it is easy to verify that the other claim in this case holds.

The approximation algorithm for rings.

LEMMA 4.21. Let $N, \varepsilon$ and $\delta$ be parameters as above, $\mathcal{B}$ be a set of $m$ weighted ( $\delta$-large) blocks with vertices in $\llbracket N \rrbracket^{2}$, and let $k$ be a parameter. Let $Q$ be the set of all possible rings with at most $k$ vertices within $\llbracket N \rrbracket^{2}$. For a ring $\sigma \in Q$, let $W_{\mathrm{opt}}(T)$ be the weight of the maximum weight independent set of blocks of $\mathcal{B}$. Then, one can $(1-\varepsilon)$-approximate $W_{\text {opt }}(\cdot)$ for all the trails of $Q$. The running time of the algorithm is $O\left(N^{O(k / \varepsilon)} m\right)$.

Proof. Using the algorithm of Lemma 4.21, we compute the maximum weight independent set for each possible trail with $O(k / \varepsilon)$ vertices within $\llbracket N \rrbracket^{2}$. Now, we compute a set $Q$ of all possible 
rings within $\llbracket N \rrbracket^{2}$ with at most $k+O(1)$ vertices each. We try all possible partitions of the rings as described by Lemma 4.20. The only difference from the algorithm of Lemma 4.21 is observing that case (C) of Lemma 4.20 corresponds to breaking a ring into a trail/ring, by taking only the first trail in the decomposition. Clearly, repeating this choice multiple times would yield the desired approximation.

\subsection{The Result}

\subsubsection{For Blocks.}

Lemma 4.22. Given $N, \varepsilon$ and $\delta$ as above, and a set $\mathcal{B}$ of $m$ weighted blocks contained in the square $[0, N]^{2}$, one can $(1-\varepsilon)$-approximate a maximum weight independent set of blocks in $\mathcal{B}$ in $O\left(N^{1 /(\varepsilon \delta)^{O(1)}} m\right)$ time.

Proof. Let $O$ be the maximum weight independent set of blocks of $\mathcal{B}$. By Lemma $4.17_{\mathrm{p} 32}$, there exists a partition of $[0, N]^{2}$ into a collection of $O\left(1 /\left(\delta \varepsilon^{2}\right)\right)$ faces $\mathcal{F}_{+}$and possibly empty area, where each face in $\mathcal{F}_{+}$is either a trail or a ring, with $\left(\frac{1}{\varepsilon \cdot \delta}\right)^{4}$ vertices. Furthermore, there is subset $O^{\prime} \subseteq O$, of weight $\geq(1-\varepsilon / 4) w(O)$, such that each block of $O^{\prime}$ is fully contained in some face of $\mathcal{F}_{+}$.

As such, we enumerate all such possible partitions. There are $N^{1 /(\varepsilon \delta)^{O(1)}}$ of them. For each such partition, and each face of the partition, we apply the $(1-\varepsilon / 4)$-approximation algorithm of Lemma 4.19 to each face that is a trail, and the algorithm of Lemma 4.21 if it is a ring. In both cases, the algorithm is run with the subset of blocks of $\mathcal{B}$ contained in the face.

Clearly, this yields the desired approximation with the desired running time.

\subsubsection{For Rectangles.}

Theorem 4.23. Given a positive integer $N>0$, parameters $\varepsilon>0$ and $\delta>0$, and a set $\mathcal{R}$ of $m$ weighted rectangles, such that the vertices of all rectangles belong to $\llbracket N \rrbracket^{2}$, where $\llbracket N \rrbracket=\{0, \ldots, N\}$. Assume that each rectangle in $\mathcal{R}$ is $\delta$-large; that is, either its height or its width is larger than $\delta N$. For this input, there is a $(1-\varepsilon)$-approximation algorithm for maximum weight independent set of rectangles with a running time of $O\left((m N)^{1 /(\varepsilon \delta)^{O(1)}}\right)$.

Proof. Let $O \subseteq \mathcal{R}$ be the optimal solution. For every rectangle $\mathrm{r} \in \mathcal{R}$, pick an arbitrary block $\mathrm{b}_{\mathrm{r}} \subseteq \mathrm{r}$, such that $\mathrm{b}_{\mathrm{r}}$ is parallel to the longer edge of $\mathrm{r}$, and set $w(\mathrm{~b})=w(\mathrm{r})$. Let $\mathcal{B}_{\text {opt }}$ be the resulting set of blocks (for the set $O$ ).

For a trail or a ring $\sigma$, a rectangle $r \in \mathcal{R}$ interacts with $\sigma$ if $r$ intersects the boundary of $\sigma$ but $\mathrm{b}_{\mathrm{r}}$ does not. Let $I(\sigma)$ denote the set of rectangles of $O$ that interact with $\sigma$. Note that during the execution of the algorithm of Lemma 4.22 (on the set $\mathcal{B}$ with the solution $\mathcal{B}_{\text {opt }}$ ), all the rings and trails considered have at most $1 /(\varepsilon \delta)^{O(1)}$ vertices.

So consider such a narrow polygon $\sigma$, and observe that $|I(\sigma)| \leq 1 /(\varepsilon \delta)^{O(1)}$. Indeed, either a vertex of $\sigma$ is covered by a rectangle $r \in O$, or alternatively, the boundary of $\sigma$ enters $r$ through one of its short edges, and leave through the other (as otherwise, $b_{r}$ would intersect the boundary of $\sigma$ ). But then, at least the portion of the boundary of $\sigma$ covered by $\mathrm{r}$ is at least $\delta N$, and as $\sigma$ 's boundary has length at most $1 /(\varepsilon \delta)^{O(1)} N$, it follows that the number of such rectangles in $O$, since all the rectangles in $O$ are disjoint, is bounded by $\frac{1 /(\varepsilon \delta)^{O(1)} N}{\delta N}=1 /(\varepsilon \delta)^{O(1)}$.

This suggest the following. Run the algorithm of Lemma 4.22 on $\mathcal{B}$ - it enumerates hierarchically over partitions of the input square. For every ring or trail $\sigma$ considered by this algorithm, guess the associated set of rectangles (of the optimal solution) it interacts with. Now, when considering a partition of such a polygon into two subpolygons, we also need to keep track of these sets of rectangles for the two subpolygons, and make sure they are maintained correctly during the dynamic programming. 
By the correctness of Lemma 4.22, one of the considered partitions rejects rectangles with total weight $\leq \varepsilon w\left(\mathcal{B}_{\text {opt }}\right)=\varepsilon w(O)$. Since there are $m^{1 /(\varepsilon \delta)^{O(1)}}$ possible interacting subsets, it follows that the new approximation algorithm has running time $(m N)^{1 /(\varepsilon \delta)^{O(1)}}$ and yields the desired approximation.

The above algorithm has pseudo-polynomial running time, since it depends polynomially on $N$. Next, we give a reduction of an instance of $\delta$-large rectangles with arbitrary $N$ to an instance of $\delta / 8$-large rectangles where $N$ is bounded by a polynomial in $m$ and $\delta$.

LEMMA 4.24. Given an integer $N>0$, a value $\delta>0$, and a set $\mathcal{R}$ of $m$ weighted rectangles such that all vertices belong to $\llbracket N \rrbracket^{2}$ and each rectangle is $\delta$-large. Then there is a set $\overline{\mathcal{R}}$ of $m$ weighted rectangles such that all vertices belong to $\llbracket \bar{N} \rrbracket^{2}$ where $\bar{N}=O(m / \delta)$, each rectangle in $\overline{\mathcal{R}}$ is $\delta / 8$-large, and there is a bijection $f: \mathcal{R} \rightarrow \overline{\mathcal{R}}$ such that for each rectangle $r \in \mathcal{R}$ the weight of $f(\mathrm{r})$ equals the weight of $\mathrm{r}$ and any set $\mathcal{R}^{\prime} \subseteq \mathcal{R}$ is independent if and only if $f\left(\mathcal{R}^{\prime}\right)$ is independent.

Proof. Let $\delta^{\prime}>0$ be the largest positive value such that $\delta^{\prime} \leq \delta$ and $1 / \delta^{\prime}$ is an integer. We have that $\delta^{\prime} \geq \delta / 2$. Define $\bar{N}:=8 \mathrm{~m} / \delta^{\prime}$. For each rectangle $\mathrm{r}_{i} \in \mathcal{R}$, we define a rectangle $f\left(\mathrm{r}_{i}\right) \in \overline{\mathcal{R}}$ with the same weight as $\mathrm{r}_{i}$. Assume that $\mathrm{r}_{i}=\left(x_{i}, x_{i}^{\prime}\right) \times\left(y_{i}, y_{i}^{\prime}\right)$. For each $\mathrm{r}_{i} \in \mathcal{R}$, we define values $\bar{x}_{i}, \bar{x}_{i}^{\prime}, \bar{y}_{i}, \bar{y}_{i}^{\prime}$ and then set $f\left(\mathrm{r}_{i}\right):=\left(\bar{x}_{i}, \bar{x}_{i}^{\prime}\right) \times\left(\bar{y}_{i}, \bar{y}_{i}^{\prime}\right)$.

For each $k \in\left\{0, \ldots, 4 / \delta^{\prime}-1\right\}$ we do the following. Let $\mathcal{R}_{k}^{L}$ denote all rectangles $\mathrm{r}_{i} \in \mathcal{R}$ such that $x_{i} \in\left[k \cdot \frac{\delta^{\prime}}{4}(N+1),(k+1) \cdot \frac{\delta^{\prime}}{4}(N+1)\right)$ and let $\mathcal{R}_{k}^{R}$ denote all rectangles $\mathrm{r}_{i} \in \mathcal{R}$ such that $x_{i}^{\prime} \in$ $\left[k \cdot \frac{\delta^{\prime}}{4}(N+1),(k+1) \cdot \frac{\delta^{\prime}}{4}(N+1)\right)$. We define the coordinate $\bar{x}_{i}$ for each $f\left(r_{i}\right)$ with $\mathrm{r}_{i} \in \mathcal{R}_{k}^{L}$ and the coordinate $\bar{x}_{i}^{\prime}$ for each $f\left(\mathrm{r}_{i}\right)$ with $\mathrm{r}_{i} \in \mathcal{R}_{k}^{R}$ such that all these coordinates are integers in the range $\left\{k \cdot \frac{\delta^{\prime}}{4} \bar{N}, \ldots, k \cdot \frac{\delta^{\prime}}{4} \bar{N}+2 m-1\right\}$ and such that their relative order is the same as the relative order as the respective coordinates $x_{i}, x_{i}^{\prime}$, i.e., for any $\mathrm{r}_{i}, \mathrm{r}_{i^{\prime}} \in \mathcal{R}_{k}^{L}$ we have that $\bar{x}_{i} \leq \bar{x}_{i^{\prime}}$ if and only if $x_{i} \leq x_{i^{\prime}}$, for any $\mathrm{r}_{i}, \mathrm{r}_{i^{\prime}} \in \mathcal{R}_{k}^{R}$ we have that $\bar{x}_{i}^{\prime} \leq \bar{x}_{i^{\prime}}^{\prime}$ if and only if $x_{i}^{\prime} \leq x_{i^{\prime}}^{\prime}$, and for any $\mathrm{r}_{i} \in \mathcal{R}_{k}^{L}, \mathrm{r}_{i^{\prime}} \in \mathcal{R}_{k}^{R}$ we have that $\bar{x}_{i} \leq \bar{x}_{i^{\prime}}^{\prime}$ if and only if $x_{i} \leq x_{i^{\prime}}^{\prime}$. It is possible to find such coordinates, since there are $2 m$ values available and all rectangles together have only $2 m$ vertical edges.

We do a symmetric procedure for the $y$-coordinates of all rectangles. We want to prove now that the resulting rectangles are $\delta^{\prime} / 4$-large. If a rectangle $\mathrm{r}_{i} \in \mathcal{R}$ is $\delta$-large, then it is also $\delta^{\prime}$ large. Assume that its width is larger than $\delta^{\prime} N$, i.e., $x_{i}^{\prime}-x_{i}>\delta^{\prime} N$. Then there must be a value $k \in\left\{0, \ldots, 4 / \delta^{\prime}-1\right\}$ such that $x_{i}<k \cdot \frac{\delta^{\prime}}{4}(N+1)$ and $x_{i}^{\prime} \geq(k+1) \cdot \frac{\delta^{\prime}}{4}(N+1)$. Hence, $\bar{x}_{i}<k \cdot \frac{\delta^{\prime}}{4} \bar{N}$ and $\bar{x}_{i}^{\prime} \geq(k+1) \cdot \frac{\delta^{\prime}}{4} \bar{N}$. This implies that $\bar{x}_{i}^{\prime}-\bar{x}_{i} \geq \frac{\delta^{\prime}}{4} \bar{N}$ and therefore $f\left(\mathrm{r}_{i}\right)$ is $\delta^{\prime} / 4$-large and also $\delta / 8$-large. Since we kept the relative order of the changed coordinates, we have that any set $\mathcal{R}^{\prime} \subseteq \mathcal{R}$ is independent if and only if $f\left(\mathcal{R}^{\prime}\right)$ is independent.

Now Lemma 4.24 and Theorem 4.23 imply our main theorem in this section.

Theorem 4.25. Given a positive integer $N>0$, parameters $\varepsilon>0$ and $\delta>0$, and a set $\mathcal{R}$ of $m$ weighted rectangles, such that the vertices of all rectangles belong to $\llbracket N \rrbracket^{2}$, where $\llbracket N \rrbracket=\{0, \ldots, N\}$. Assume that each rectangle in $\mathcal{R}$ is $\delta$-large; that is, either its height or its width is larger than $\delta N$. For this input, there is a $(1-\varepsilon)$-approximation algorithm for maximum weight independent set of rectangles with a running time of $O\left((m / \delta)^{1 /(\varepsilon \delta)^{O(1)}}\right)$.

\section{CONCLUSIONS}

We presented a QPTAS for the maximum independent set of polygons problem. Contrasting this, the best-known approximation algorithm with polynomial running time has a performance ratio of $n^{\varepsilon}$. Furthermore, even for the axis-parallel rectangles case no constant factor approximation 
algorithm in polynomial time is known. Our QPTAS suggests that better polynomial time approximation algorithms are possible. In particular, our PTAS for the case of large rectangles might well turn out to be a first step toward a PTAS for the general case.

For our results, we presented two new techniques: the recursive partitioning that paved the way to our QPTAS and the partition into $O(1)$ thin corridors and cycles in our PTAS. Soon after first publishing our results these techniques were used for other geometric problems, see Section 1.3. We believe that there will be more applications of them in other geometric settings.

Recently, Chuzhoy and Ene presented a $(1+\varepsilon)$-approximation algorithm for independent set of (unweighted) Rectangles with a running time of $n^{\text {poly }(\log \log n / \varepsilon)}[15]$ by building on the methodology presented in this article and significantly extending it.

\section{ACKNOWLEDGMENTS}

The authors thank Chandra Chekuri, János Pach, and Kasturi Varadarajan for useful discussions on the problems studied in this article.

\section{REFERENCES}

[1] A. Anagnostopoulos, F. Grandoni, S. Leonardi, and A. Wiese. 2013. Constant integrality gap LP formulations of unsplittable flow on a path. In Proceedings of the 16th Conference on Integer Programming and Combinatorial Optimization (IPCO). 25-36. DOI : https://doi.org/10.1007/978-3-642-36694-9_3.

[2] P. K. Agarwal, M. van Kreveld, and S. Suri. 1998. Label placement by maximum independent set in rectangles. Comput. Geom. Theory Appl. 11 (1998), 209-218.

[3] P. K. Agarwal and N. H. Mustafa. 2006. Independent set of intersection graphs of convex objects in 2D. Comput. Geom. Theory Appl. 34, 2 (2006), 83-95.

[4] S. Arora. 1998. Polynomial time approximation schemes for Euclidean TSP and other geometric problems. F. Assoc. Comput. Mach. 45, 5 (Sept. 1998), 753-782.

[5] A. Adamaszek and A. Wiese. 2013. Approximation schemes for maximum weight independent set of rectangles. In Proceedings of the 54th Annual IEEE Symposium on Foundations of Computer Science (FOCS). 400-409.

[6] A. Adamaszek and A. Wiese. 2014. A QPTAS for maximum weight independent set of polygons with polylogarithmic many vertices. In Proceedings of the 25th ACM-SIAM Symposium on Discrete Algorithms (SODA). 400-409.

[7] A. Adamaszek and A. Wiese. 2015. A quasi-PTAS for the two-dimensional geometric knapsack problem. In Proceedings of the 26th ACM-SIAM Symposium on Discrete Algorithms (SODA'15). SIAM, 1491-1505. DOI : https://doi.org/10.1137/ 1.9781611973730 .98

[8] S. Bandyapadhyay, S. Bhowmick, and K. R. Varadarajan. 2015. Approximation schemes for partitioning: Convex decomposition and surface approximation. In Proceedings of the 26th ACM-SIAM Symposium on Discrete Algorithms (SODA'15). SIAM, 1457-1470. DOI : https://doi.org/10.1137/1.9781611973730.96

[9] P. Berman, B. DasGupta, S. Muthukrishnan, and S. Ramaswami. 2001. Efficient approximation algorithms for tiling and packing problems with rectangles. F. Algor. 41, 2 (2001), 443-470.

[10] P. Berman and T. Fujito. 1999. On approximation properties of the independent set problem for low degree graphs. Theo. Comp. Sci. 32, 2 (1999), 115-132.

[11] M. de Berg and O. Schwarzkopf. 1995. Cuttings and applications. Int. f. Comput. Geom. Appl. 5, 4 (1995), 343-355. DOI : $10.1142 / S 0218195995000210$

[12] P. Bonsma, J. Schulz, and A. Wiese. 2011. A constant factor approximation algorithm for unsplittable flow on paths. In Proceedings of the 52nd Annual IEEE Symposium on Foundations of Computer Science (FOCS). 47-56. DOI : https:// doi.org/10.1109/FOCS.2011.10

[13] P. Chalermsook and J. Chuzhoy. 2009. Maximum independent set of rectangles. In Proceedings of the 20th ACM-SIAM Symposium on Discrete Algorithms (SODA). 892-901.

[14] B. N. Clark, C. J. Colbourn, and D. S. Johnson. 1990. Unit disk graphs. Discr. Math. 86, 1-3 (1990), 165-177. DOI : 10. 1016/0012-365X(90)90358-O

[15] J. Chuzhoy and A. Ene. 2016. On approximating maximum independent set of rectangles. In Proceedings of the 57th Annual IEEE Symposium on Foundations of Computer Science (FOCS'16). 820-829. DOI : https://doi.org/10.1109/FOCS. 2016.92

[16] B. Chazelle and J. Friedman. 1990. A deterministic view of random sampling and its use in geometry. Combinatorica 10, 3 (1990), 229-249. 
[17] T. M. Chan and S. Har-Peled. 2012. Approximation algorithms for maximum independent set of pseudo-disks. Discr. Comput. Geom. 48, 2 (2012), 373-392. DOI : 10.1007/s00454-012-9417-5

[18] B. Chazelle. 1993. Cutting hyperplanes for divide-and-conquer. Discr. Comput. Geom. 9, 2 (1993), 145-158.

[19] T. M. Chan. 2003. Polynomial-time approximation schemes for packing and piercing fat objects. F. Algor. 46, 2 (2003), 178-189.

[20] T. M. Chan. 2004. Euclidean bounded-degree spanning tree ratios. Discrete and Computational Geometry 32, 2 (2004), 177-194. http://www.springerlink.com/index/10.1007/s00454-004-1117-3.

[21] K. L. Clarkson and P. W. Shor. 1989. Applications of random sampling in computational geometry, II. Discr. Comput. Geom. 4, 5 (1989), 387-421. DOI : 10.1007/BF02187740

[22] L. de Floriani, P. Magillo, and Puppo E. 2000. Applications of computational geometry to geographic information systems. In Handbook of Computational Geometry, J.-R. Sack and J. Urrutia (Eds.). North Holland, Amsterdam, 333388.

[23] A. Ene, S. Har-Peled, and B. Raichel. 2012. Geometric packing under non-uniform constraints. In Proceedings of the 28th Annual Symposium on Computational Geometry (SoCG). 11-20.

[24] T. Erlebach, K. Jansen, and E. Seidel. 2005. Polynomial-time approximation schemes for geometric intersection graphs. SIAM f. Comput. 34, 6 (2005), 1302-1323.

[25] T. Fukuda, Y. Morimoto, S. Morishita, and T. Tokuyama. 2001. Data mining with optimized two-dimensional association rules. ACM Trans. Data. Sys. 26, 2 (2001), 179-213.

[26] J. Fox and J. Pach. 2008. Separator theorems and Turán-type results for planar intersection graphs. Adv. Math. 219, 3 (2008), 1070-1080.

[27] J. Fox and J. Pach. 2011. Computing the independence number of intersection graphs. In Proceedings of the 22nd ACM-SIAM Symposium on Discrete Algorithms (SODA). 1161-1165.

[28] R. J. Fowler, M. S. Paterson, and S. L. Tanimoto. 1981. Optimal packing and covering in the plane are NP-complete. Inform. Process. Lett. 12, 3 (1981), 133-137.

[29] S. Har-Peled. 2000. Constructing planar cuttings in theory and practice. SIAM J. Comput. 29, 6 (2000), 2016-2039.

[30] S. Har-Peled. 2009. Being fat and friendly is not enough. CoRR abs/0908.2369 (2009).

[31] S. Har-Peled. 2011. Geometric Approximation Algorithms. Mathematical Surveys and Monographs, Vol. 173. American Mathematical Society, Boston, MA.

[32] S. Har-Peled. 2014. Quasi-polynomial time approximation scheme for sparse subsets of polygons. In Proceedings of the 30th Annual Symposium on Computational Geometry (SoCG'14). 120-129. DOI : https://doi.org/10.1145/2582112. 2582157

[33] S. Har-Peled. 2016. Shortest path in a polygon using sublinear space. f. Comput. Geom. 7, 2 (2016), 19-45. http://jocg. org/index.php/jocg/article/view/256.

[34] D. S. Hochbaum and W. Maas. 1985. Approximation schemes for covering and packing problems in image processing and VLSI. F. Assoc. Comput. Mach. 32, 1 (1985), 130-136. https://doi.org/10.1145/2455.214106

[35] J. Hershberger and S. Suri. 1995. A pedestrian approach to ray shooting: Shoot a ray, take a walk. f. Algor. 18, 3 (1995), 403-431.

[36] H. Imai and T. Asano. 1983. Finding the connected components and a maximum clique of an intersection graph of rectangles in the plane. F. Algor. 4, 4 (1983), 310-323.

[37] Marek Karpinski, Andrzej Lingas, and Dzmitry Sledneu. 2018. A QPTAS for the base of the number of triangulations of a planar point set. Theor. Comput. Sci. 711 (2018), 56-65. DOI : 10.1016/j.tcs.2017.11.003

[38] S. Khanna, S. Muthukrishnan, and M. Paterson. 1998. On approximating rectangle tiling and packing. In Proceedings of the 9th ACM-SIAM Symposium on Discrete Algorithms (SODA). 384-393.

[39] L. Lewin-Eytan, J. Naor, and A. Orda. 2002. Routing and admission control in networks with advance reservations. In Proceedings of the 5th International Conference on Approximation Algorithms for Combinatorial Optimization Problems (APPROX'02), Lecture Notes in Computer Science, Vol. 2462. 215-228.

[40] B. Lent, A. Swami, and J. Widom. 1997. Clustering association rules. In Proceedings of the 13th IEEE International Conference on Data Engineering. IEEE, 220-231. DOI : https://doi.org/10.1109/ICDE.1997.581756

[41] R. J. Lipton and R. E. Tarjan. 1979. A separator theorem for planar graphs. SIAM J. Appl. Math. 36 (1979), 177-189.

[42] J. Matoušek. 2014. Near-optimal separators in string graphs. Combin. Prob. Comput. 23, 1 (2014), 135-139. DOI : https:// doi.org/10.1017/S0963548313000400

[43] G. L. Miller. 1986. Finding small simple cycle separators for 2-connected planar graphs. 7. Comput. Sys. Sci. 32, 3 (1986), 265-279.

[44] D. Marx and M. Pilipczuk. 2015. Optimal parameterized algorithms for planar facility location problems using voronoi diagrams. In Proceedings of the 23nd Annual Euro. Symposium Algorithms (ESA). Lecture Notes in Computer Science, Vol. 9294. Springer, Berlin, 865-877. DOI : https://doi.org/10.1007/978-3-662-48350-3_72 
[45] N. H. Mustafa, R. Raman, and S. Ray. 2014. Settling the APX-Hardness status for geometric set cover. In Proceedings of the 55th IEEE Annual Symposium on Foundations of Computer Science (FOCS'14). IEEE Computer Society, 541-550. https://doi.org/10.1109/FOCS.2014.64

[46] F. Nielsen. 2000. Fast stabbing of boxes in high dimensions. Theo. Comp. Sci. 246, 1-2 (2000), 53-72. DOI : https:// doi.org/10.1016/S0304-3975(98)00336-3

[47] Giorgi Nadiradze and Andreas Wiese. 2016. On approximating strip packing with a better ratio than 3/2. In Proceedings of the 27th ACM-SIAM Symposium on Discrete Algorithms (SODA'16). SIAM, 1491-1510. DOI : https://doi.org/10.1137/ 1.9781611974331.ch102

[48] M. Sharir and P. K. Agarwal. 1995. Davenport-Schinzel Sequences and Their Geometric Applications. Cambridge University Press, New York.

[49] M. Sharir. 2003. The Clarkson-Shor technique revisited and extended. Combin. Prob. Comput. 12, 2 (2003), $191-201$.

[50] W. D. Smith and N. C. Wormald. 1998. Geometric separator theorems and applications. In Proceedings of the 39th Annual IEEE Symposium on Foundations of Computer Science (FOCS). 232-243.

[51] B. Verweij and K. Aardal. 1999. An optimisation algorithm for maximum independent set with applications in map labelling. In Proceedings of the 7th Annual European Symposium Algorithms (ESA). 426-437.

[52] D. Zuckerman. 2007. Linear degree extractors and the inapproximability of max clique and chromatic number. Theory Comput. 3, 6 (2007), 103-128. DOI : https://doi.org/10.4086/toc.2007.v003a006

Received March 2018; revised March 2019; accepted April 2019 